quatrième série-tome $44 \quad$ fascicule $4 \quad$ juillet-août 2011

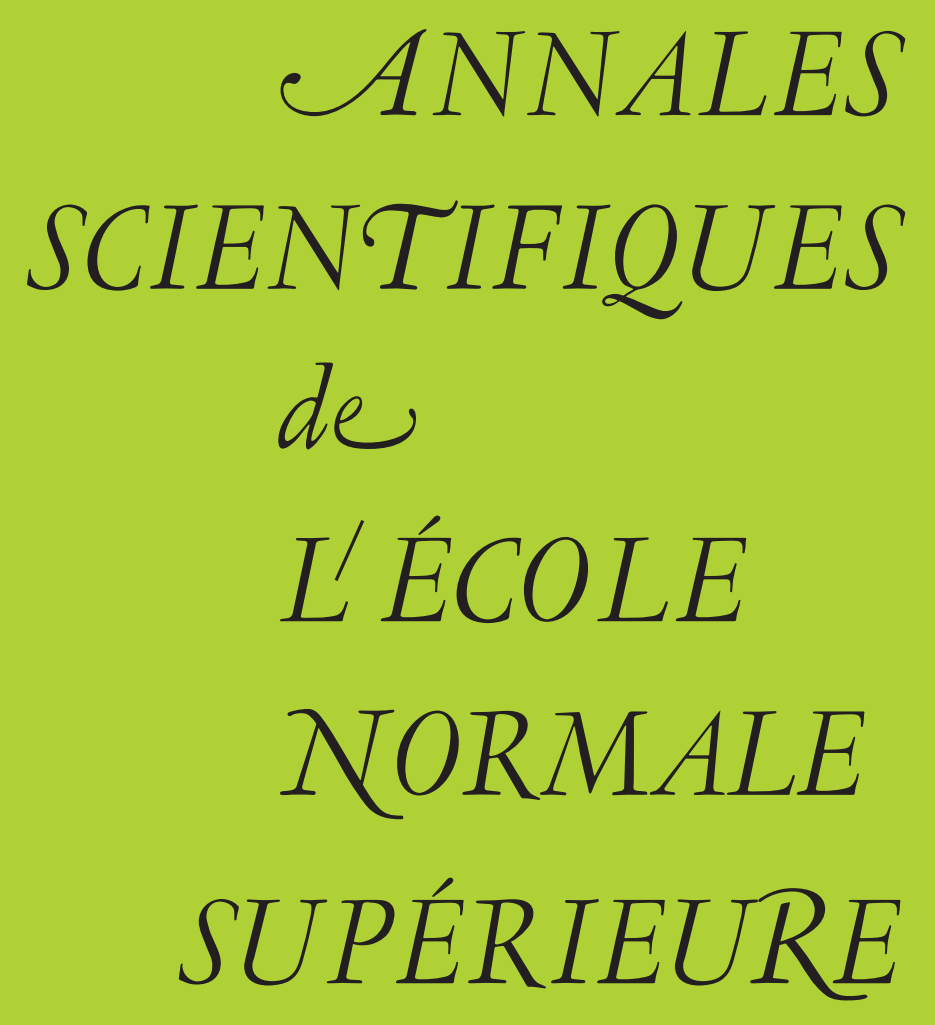

Sébastien BLACHÈRE \& Peter HAÏSSINSKY \& Pierre MATHIEU Harmonic measures versus quasiconformal measures for byperbolic groups 
Ann. Scient. Éc. Norm. Sup.

$4^{\mathrm{e}}$ série, t. 44, 2011, p. 683 à 721

\title{
HARMONIC MEASURES VERSUS QUASICONFORMAL MEASURES FOR HYPERBOLIC GROUPS
}

\author{
BY Sébastien BLACHÈRE, Peter HAÏSSINSKY \\ AND PIERRE MATHIEU
}

\begin{abstract}
We establish a dimension formula for the harmonic measure of a finitely supported and symmetric random walk on a hyperbolic group. We also characterize random walks for which this dimension is maximal. Our approach is based on the Green metric, a metric which provides a geometric point of view on random walks and, in particular, which allows us to interpret harmonic measures as quasiconformal measures on the boundary of the group.

RÉsumé. - On établit une formule de la dimension de la mesure harmonique d'une marche aléatoire de loi de support fini et symétrique sur un groupe hyperbolique. On caractérise aussi les lois pour lesquelles la dimension est maximale. Notre approche repose sur la distance de Green, une distance qui permet de développer un point de vue géométrique sur les marches aléatoires et, en particulier, d'interpréter les mesures harmoniques comme des mesures quasiconformes.
\end{abstract}

\section{Introduction}

Let $\Gamma$ be a non-elementary word hyperbolic group. There are two main constructions of measures on the boundary $\partial \Gamma$ of $\Gamma$ : quasiconformal measures and harmonic measures. Let us recall these constructions.

Given a cocompact properly discontinuous action of $\Gamma$ by isometries on a pointed proper geodesic metric space $(X, w, d)$, the Patterson-Sullivan procedure consists in taking weak limits of

$$
\frac{1}{\sum_{\gamma \in \Gamma} e^{-s d(w, \gamma(w))}} \sum_{\gamma \in \Gamma} e^{-s d(w, \gamma(w))} \delta_{\gamma(w)}
$$

as $s$ decreases to the logarithmic volume growth

$$
v \stackrel{\text { def }}{=} \limsup _{R \rightarrow \infty} \frac{1}{R} \log |B(w, R) \cap \Gamma(w)| .
$$

Patterson-Sullivan measures are quasiconformal measures and Hausdorff measures of $\partial X$ when endowed with a visual metric. 
Given a probability measure $\mu$ on $\Gamma$, the random walk $\left(Z_{n}\right)_{n}$ starting from the neutral element $e$ associated with $\mu$ is defined by

$$
Z_{0}=e ; Z_{n+1}=Z_{n} \cdot X_{n+1},
$$

where $\left(X_{n}\right)$ is a sequence of independent and identically distributed random variables of law $\mu$. Under some mild assumptions on $\mu$, the walk $\left(Z_{n}\right)_{n}$ almost surely converges to a point $Z_{\infty} \in \partial \Gamma$. The law of $Z_{\infty}$ is by definition the harmonic measure $\nu$.

The purpose of this work is to investigate the interplay between these two classes of measures and take advantage of this interplay to derive information on the geometry of harmonic measures.

We show that, for a general hyperbolic group, the Hausdorff dimension of the harmonic measure satisfies a 'dimension-entropy-rate of escape' formula and we characterize those harmonic measures of maximal dimension. These results are intimately connected with a sharp control of the deviation of sample paths of the random walk with respect to geodesics.

Not surprisingly, the starting point is A. Ancona's theorem stating that the Green function is almost multiplicative along geodesics [2, Thm 6.1].

The usual tool for studying selfsimilar measures is to replace the action of the group by a linear-in-time action of a dynamical system and then to apply the thermodynamic formalism to it. Indeed it is tempting to interpret Ancona's estimate as a kind of Gibbs property of the harmonic measure. This strategy was successfully used in special cases: for free groups and Fuchsian groups, a Markov-map $F_{\Gamma}$ has been introduced on the boundary which is orbit-equivalent to $\Gamma[12,35]$. For discrete subgroups of isometries of a Cartan-Hadamard manifold, one may work with the geodesic flow [24, 26, 33, 34]. Both these methods seem difficult to implement for general hyperbolic groups. On the one hand, it is not obvious how to associate a Markov map with a general hyperbolic group; in particular, not enough is known on the automatic structure of a hyperbolic group to derive a coding of its boundary. On the other hand, the construction of the geodesic flow for general hyperbolic spaces is delicate and its mixing properties do not seem strong enough to apply the thermodynamic formalism.

Our approach is completely different: it directly combines geometric and probabilistic arguments without any reference to the thermodynamic formalism. We make a heavy use of the so-called Green metric associated with the random walk. Ancona's theorem implies that the Green metric is hyperbolic in the sense of Gromov. It is then a simple but crucial observation that the harmonic measure is actually a Patterson-Sullivan measure. Hence the Green metric provides us with a genuine geometric framework for random walks. The computation of the dimension of the harmonic measure now follows from a straightforward argument. The explicit expression of the Green metric in terms of the hitting probability of the random walk makes it possible to directly take advantage of the independence of the increments of the walk. The combination of both facts - hyperbolicity and independenceyields very precise estimates on how random paths deviate from geodesics. This is used for analyzing the harmonic measures of maximal dimension. We also get an alternative and rather straightforward proof of the fact that the harmonic measure of a random walk on a Fuchsian group with cusps is singular, a result previously established in [19] and [15] by completely different methods. 
The Green metric is not geodesic in general, so that we are led to developing a new framework for a special class of non-geodesic hyperbolic spaces that we call quasiruled spaces. Thus, we extend classical results such as the approximation of finite configurations by trees and the theory of quasiconformal measures.

We believe that our approach is also significant from a purely geometric point of view. For instance, it raises the question whether-in general-Patterson-Sullivan measures of maximal Hausdorff dimension can be similarly characterized. To our knowledge, this problem is completely open; compare with [13].

The rest of this introduction is devoted to a more detailed description of our results.

\subsection{Geometric setting}

Given a hyperbolic group $\Gamma$, we let $\mathscr{D}(\Gamma)$ denote the collection of hyperbolic left-invariant metrics on $\Gamma$ which are quasi-isometric to a word metric induced by a finite generating set of $\Gamma$. In general these metrics do not come from proper geodesic metric spaces as we will see (cf. Theorem 1.1 for instance). In the sequel, we will distinguish the group as a space and as acting on a space: we keep the notation $\Gamma$ for the group, and we denote by $X$ the group as a metric space endowed with a metric $d \in \mathscr{D}(\Gamma)$. We may equivalently write $(X, d) \in \mathscr{D}(\Gamma)$. We will often require a base point which we will denote by $w \in X$. We recall that the boundary can be supplied with a family of so-called visual distances $d_{\varepsilon}$ which essentially depend on a visual parameter $\varepsilon>0$ (cf. §2).

This setting enables us to capture in particular the following two situations.

- Assume that $\Gamma$ admits a cocompact properly discontinuous action by isometries on a proper geodesic space $(Y, d)$. Pick $w \in Y$ such that $\gamma \in \Gamma \mapsto \gamma(w)$ is a bijection, and consider $X=\Gamma(w)$ with the restriction of $d$.

- We may choose $(X, d)=\left(\Gamma, d_{G}\right)$ where $d_{G}$ is the Green metric associated with a random walk (see Theorem 1.1).

Let $\mu$ be a symmetric probability measure the support of which generates $\Gamma$. Even if the support of $\mu$ may be infinite, we will require some compatibility with the geometry of the quasi-isometry class of $\mathscr{D}(\Gamma)$. Thus, we will often assume one of the following two assumptions. Given a metric $(X, d) \in \mathscr{D}(\Gamma)$, we say that the random walk has finite first moment if

$$
\sum_{\gamma \in \Gamma} d(w, \gamma(w)) \mu(\gamma)<\infty
$$

We say that the random walk has an exponential moment if there exists $\lambda>0$ such that

$$
\sum_{\gamma \in \Gamma} e^{\lambda d(w, \gamma(w))} \mu(\gamma)<\infty
$$

Note that both these conditions only depend on the quasi-isometry class of the metric. 


\subsection{The Green metric}

The analogy between both families of measures - quasiconformal and harmonic - has already been pointed out in the literature e.g. [13, 26, 36]. Our first task is to make this empirical fact a theorem, i.e., we prove that harmonic measures are indeed quasiconformal measures for a well-chosen metric: given a symmetric law $\mu$ on $\Gamma$ such that its support generates $\Gamma$, let $F(x, y)$ be the probability that the random walk started at $x$ ever hits $y$, i.e., the probability there is some $n$ such that $x Z_{n}=y$. Let

$$
d_{G}(x, y) \stackrel{\text { def }}{=}-\log F(x, y) .
$$

This function $d_{G}$ is known to be a left-invariant metric on $\Gamma$. It was first introduced by $\mathrm{S}$. Blachère and S. Brofferio in [8] and further studied in [9]. It is non-degenerate as soon as the walk is transient, i.e., eventually leaves any finite set. This is the case as soon as $\Gamma$ is a non-elementary hyperbolic group.

The Green function is defined by

$$
G(x, y) \stackrel{\text { def }}{=} \sum_{n=0}^{\infty} \mathbb{P}\left[x Z_{n}=y\right]=\sum_{n=0}^{\infty} \mu^{n}\left(x^{-1} y\right),
$$

where, for each $n \geq 1, \mu^{n}$ is the law of $Z_{n}$, i.e., the $n$th convolution power of the measure $\mu$. The Markov property and the invariance by the group imply

$$
G(x, y)=F(x, y) G(y, y)=F(x, y) G(e, e)
$$

so that

$$
d_{G}(x, y)=\log G(e, e)-\log G(x, y) .
$$

Non-elementary hyperbolic groups are non-amenable and for such groups and finitely supported laws $\mu$, it was proved in [8] that the Green and word metrics are quasi-isometric. Nevertheless it does not follow from this simple fact that $d_{G}$ is hyperbolic.

We first prove the following:

THEOREM 1.1. - Let $\Gamma$ be a non-elementary hyperbolic group, $\mu$ a symmetric probability measure on $\Gamma$ the support of which generates $\Gamma$.

(i) Assume that $\mu$ has an exponential moment, then $d_{G} \in \mathscr{D}(\Gamma)$ if and only if for any $r$ there exists a constant $C(r)$ such that

$$
F(x, y) \leq C(r) F(x, v) F(v, y)
$$

whenever $x, y$ and $v$ are points in a locally finite Cayley graph of $\Gamma$ and $v$ is at distance at most $r$ from a geodesic segment between $x$ and $y$.

(ii) If $d_{G} \in \mathscr{D}(\Gamma)$ then the harmonic measure is Ahlfors regular of dimension $1 / \varepsilon$, when $\partial \Gamma$ is endowed with a visual metric $d_{\varepsilon}^{G}$ of parameter $\varepsilon>0$ induced by $d_{G}$.

A. Ancona proved that (1) holds for finitely supported laws $\mu$ [2, Thm 6.1] and he used this estimate as the key ingredient in proving that the Martin boundary coincides with the geometric (hyperbolic) boundary; we may track down this idea to [1], see also [26, Thm 3.1]. The relationships between the Green metric and the Martin boundary are further discussed in $\S 1.5$ and $\S 3.2$.

Theorem 1.1 in particular yields 
COROLlaRY 1.2. - Let $\Gamma$ be a non-elementary hyperbolic group, $\mu$ a finitely supported symmetric probability measure on $\Gamma$ the support of which generates $\Gamma$. Then its associated Green metric $d_{G}$ is a left-invariant hyperbolic metric on $\Gamma$ quasi-isometric to $\Gamma$ such that the harmonic measure is Ahlfors regular of dimension $1 / \varepsilon$, when $\partial \Gamma$ is endowed with a visual metric $d_{\varepsilon}^{G}$ of parameter $\varepsilon>0$ induced by $d_{G}$.

Our second source of examples of random walks satisfying (1) will come from Brownian motions on Riemannian manifolds of negative curvature. The corresponding law $\mu$ will then have infinite support (see $\S 1.6$ and $\S 6$ ).

\subsection{Dimension of the harmonic measure at infinity}

Let $(X, d) \in \mathscr{D}(\Gamma)$. We fix a base point $w \in X$ and consider the random walk on $X$ started at $w$, i.e., the sequence of $X$-valued random variables $\left(Z_{n}(w)\right)$ defined by the action of $\Gamma$ on $X$. There are (at least) two natural asymptotic quantities one can consider: the asymptotic entropy

$$
h \stackrel{\text { def }}{=} \lim _{n} \frac{-\sum_{\gamma \in \Gamma} \mu^{n}(\gamma) \log \mu^{n}(\gamma)}{n}=\lim _{n} \frac{-\sum_{x \in \Gamma(w)} \mathbb{P}\left[Z_{n}(w)=x\right] \log \mathbb{P}\left[Z_{n}(w)=x\right]}{n}
$$

which measures the way the law of $Z_{n}(w)$ is spread in different directions, and the rate of escape or drift

$$
\ell \stackrel{\text { def }}{=} \lim _{n} \frac{d\left(w, Z_{n}(w)\right)}{n}
$$

which estimates how far $Z_{n}(w)$ is from its initial point $w$. (The above limits for $h$ and $\ell$ are almost sure and in $L^{1}$ and they are finite as soon as the law has a finite first moment.)

We obtain the following.

Theorem 1.3. - Let $\Gamma$ be a non-elementary hyperbolic group, $(X, d) \in \mathscr{D}(\Gamma), d_{\varepsilon}$ be a visual metric of $\partial X$, and let $B_{\varepsilon}(a, r)$ be the ball of center $a \in \partial X$ and radius $r$ for the distance $d_{\varepsilon}$. Let $\nu$ be the harmonic measure of a random walk $\left(Z_{n}\right)$ whose increments are given by a symmetric law $\mu$ with finite first moment such that $d_{G} \in \mathscr{D}(\Gamma)$.

The pointwise Hausdorff dimension $\lim _{r \rightarrow 0} \frac{\log \nu\left(B_{\varepsilon}(a, r)\right)}{\log r}$ exists for $\nu$-almost every $a \in \partial X$, and is independent from the choice of a. More precisely, for $\nu$-almost every $a \in \partial X$,

$$
\lim _{r \rightarrow 0} \frac{\log \nu\left(B_{\varepsilon}(a, r)\right)}{\log r}=\frac{\ell_{G}}{\varepsilon \ell}
$$

where $\ell>0$ denotes the rate of escape of the walk with respect to $d$ and $\ell_{G} \stackrel{\text { def }}{=} \lim _{n} \frac{d_{G}\left(w, Z_{n}(w)\right)}{n}$ the rate of escape with respect to $d_{G}$.

We recall that the dimension of a measure is the infimum Hausdorff dimension of sets of positive measure. In [9], it was shown that $\ell_{G}=h$ the asymptotic entropy of the walk. From Theorem 1.3 , we deduce that

COROLlary 1.4. - Under the assumptions of Theorem 1.3,

$$
\operatorname{dim} \nu=\frac{h}{\varepsilon \ell}
$$

where $h$ denotes the asymptotic entropy of the walk and $\ell$ its rate of escape with respect to $d$. 
This dimension formula already appears in the work of $F$. Ledrappier for random walks on free groups [35]. See also V. Kaimanovich [27]. For general hyperbolic groups, V. Leprince established the inequality $\operatorname{dim} \nu \leq h /(\varepsilon \ell)$ and made constructions of harmonic measures with arbitrarily small dimension [31]. More recently, V. Leprince established that $h / \varepsilon \ell$ is also the box dimension of the harmonic measure under the sole assumption that the random walk has a finite first moment [32]. Note however that the notion of box dimension is too weak to ensure the existence of the pointwise Hausdorff dimension almost everywhere.

This formula is also closely related to the dimension formula proved for ergodic invariant measures with positive entropy in the context of geometric dynamical systems: the drift corresponds to a Lyapunov exponent [45].

\subsection{Characterization of harmonic measures with maximal dimension}

Given a random walk on a finitely generated group $\Gamma$ endowed with a left-invariant metric $d$, the so-called fundamental inequality between the asymptotic entropy $h$, the drift $\ell$ and the logarithmic growth rate $v$ of the action of $\Gamma$ reads

$$
h \leq \ell v \text {. }
$$

It holds as soon as all these objects are well-defined (cf. [9]). Corollary 1.4 provides a geometric interpretation of this inequality in terms of the harmonic measure: indeed, since $v / \varepsilon$ is the dimension of $\left(\partial X, d_{\varepsilon}\right)$, see [14], it is clearly larger than the dimension of $\nu$.

A. Vershik suggested the study of the case of equality (see $[18,43])$. For any hyperbolic group, Theorem 1.1 implies that the equality $h=\ell v$ holds for the Green metric and Theorem 1.5 below shows that the equality for some $d \in \mathscr{D}(\Gamma)$ implies $d$ is almost proportional to $d_{G}$. In particular, given a metric in $\mathscr{D}(\Gamma)$, all the harmonic measures for which the (fundamental) equality holds belong to the same class of quasiconformal measures.

In the sequel, two measures will be called equivalent if they share the same sets of zero measure.

Theorem 1.5. - Let $\Gamma$ be a non-elementary hyperbolic group and $(X, d) \in \mathscr{D}(\Gamma)$; let $d_{\varepsilon}$ be a visual metric of $\partial X$, and $\nu$ the harmonic measure given by a symmetric law $\mu$ with an exponential moment, the support of which generates $\Gamma$. We further assume that $\left(X, d_{G}\right) \in \mathscr{D}(\Gamma)$. We denote by $\rho$ a quasiconformal measure on $\left(\partial X, d_{\varepsilon}\right)$. The following propositions are equivalent.

(i) We have the equality $h=\ell v$ in the metric $d$.

(ii) The measures $\rho$ and $\nu$ are equivalent.

(iii) The measures $\rho$ and $\nu$ are equivalent and the density is almost surely bounded and bounded away from 0 .

(iv) The map $\left(\Gamma, d_{G}\right) \stackrel{I d}{\longrightarrow}(X, v d)$ is a $(1, C)$-quasi-isometry.

(v) The measure $\nu$ is a quasiconformal measure of $\left(\partial X, d_{\varepsilon}\right)$.

This theorem is the counterpart of a result of F. Ledrappier for Brownian motions on universal covers of compact Riemannian manifolds of negative sectional curvature [33], see also $§ 1.6$. Similar results have been established for the free group with free generators, see [35]. The case of equality $h=\ell v$ has also been studied for particular sets of generators of free products of finite groups [38]. For universal covers of finite graphs, see [36]. 
Theorem 1.5 enables us to compare random walks and decide when their harmonic measures are equivalent.

COROLlaRY 1.6. - Let $\Gamma$ be a non-elementary hyperbolic group with two finitely supported symmetric probability measures $\mu$ and $\widehat{\mu}$ where both supports generate $\Gamma$. We consider the random walks $\left(Z_{n}\right)$ and $\left(\widehat{Z}_{n}\right)$. Let us denote their Green functions by $G$ and $\widehat{G}$ respectively, the asymptotic entropies by $h$ and $\widehat{h}$, and the harmonic measures seen from the neutral element $e$ by $\nu$ and $\widehat{\nu}$. The following propositions are equivalent.

(i) We have the equality

$$
\widehat{h}=\lim \frac{-1}{n} \log G\left(e, \widehat{Z_{n}}\right)
$$

in $L^{1}$ and almost surely.

(ii) We have the equality

$$
h=\lim \frac{-1}{n} \log \widehat{G}\left(e, Z_{n}\right)
$$

in $L^{1}$ and almost surely.

(iii) The measures $\nu$ and $\widehat{\nu}$ are equivalent.

(iv) There is a constant $C$ such that

$$
\frac{1}{C} \leq \frac{G(x, y)}{\widehat{G}(x, y)} \leq C .
$$

\subsection{The Green metric and the Martin compactification}

Given a probability measure $\mu$ on a countable group $\Gamma$, one defines the Martin kernel

$$
K(x, y)=K_{y}(x) \stackrel{\text { def }}{=} \frac{G(x, y)}{G(e, y)} .
$$

By definition, the Martin compactification $\Gamma \cup \partial_{M} \Gamma$ is the smallest compactification of $\Gamma$ endowed with the discrete topology such that the Martin kernel continuously extends to $\Gamma \times\left(\Gamma \cup \partial_{M} \Gamma\right)$. Then $\partial_{M} \Gamma$ is called the Martin boundary.

A general theme is to identify the Martin boundary with a geometric boundary of the group. It was observed in [9] that the Martin compactification coincides with the Busemann compactification of $\left(\Gamma, d_{G}\right)$. We go one step further by showing that the Green metric provides a common framework for the identification of the Martin boundary with the boundary at infinity of a hyperbolic space (cf. [2, 5, 26]).

TheOREM 1.7. - Let $\Gamma$ be a countable group, $\mu$ a symmetric probability measure the support of which generates $\Gamma$. We assume that the corresponding random walk is transient. If the Green metric $d_{G}$ is hyperbolic, then the Martin boundary consists only of minimal points and it is homeomorphic to the hyperbolic boundary of $\left(\Gamma, d_{G}\right)$.

In particular, if $\Gamma$ is a non-elementary hyperbolic group and if $d_{G} \in \mathscr{D}(\Gamma)$, then $\partial_{M} \Gamma$ is homeomorphic to $\partial \Gamma$.

One easily deduces from Corollary 1.2:

Corollary 1.8 (A. Ancona). - Let $\Gamma$ be a non-elementary hyperbolic group, $\mu$ a finitely supported probability measure the support of which generates $\Gamma$. Then the Martin boundary is homeomorphic to the hyperbolic boundary of $\Gamma$. 
In $\S 6.2$, we provide examples of hyperbolic groups with random walks for which the Green metric is hyperbolic, but not in the quasi-isometry class of the group, and also examples of non-hyperbolic groups for which the Green metric is nonetheless hyperbolic. These examples are constructed by discretizing Brownian motions on Riemannian manifolds (see below).

\subsection{Brownian motion revisited}

Let $M$ be the universal covering of a compact Riemannian manifold of negative curvature with deck transformation group $\Gamma$, i.e., the action of $\Gamma$ is isometric, cocompact and properly discontinuous. The Brownian motion $\left(\xi_{t}\right)$ on $M$ is the diffusion process generated by the Laplace-Beltrami operator. The following limit exists almost surely and in $L^{1}$ and we call it the drift of the Brownian motion:

$$
\ell_{M} \stackrel{\text { def }}{=} \lim \frac{d\left(\xi_{0}, \xi_{t}\right)}{t}
$$

it is also known that $\ell_{M}>0$ and that $\left(\xi_{t}\right)$ almost surely converges to a point $\xi_{\infty}$ in $\partial M$ $[40,41]$. The distribution of $\xi_{\infty}$ is the harmonic measure. Furthermore, V. Kaimanovich has defined an asymptotic entropy $h_{M}$ which shares the same properties as for random walks [23]. He also proved that the fundamental inequality $h_{M} \leq \ell_{M} v$ remains valid in this setting, where $v$ denotes the logarithmic volume growth rate of $M$.

Refining a method of T. Lyons and D. Sullivan [37], W. Ballmann and F. Ledrappier construct in [5] a random walk on $\Gamma$ which mirrors the trajectories of the Brownian motion and to which we may apply our previous results. This enables us to recover the following results.

THeOREM 1.9. - Let $M$ be the universal covering of a compact Riemannian manifold of negative curvature. Let $v$ be the logarithmic volume growth of $M$ and $d_{\varepsilon}$ be a visual distance on $\partial M$. Then

$$
\operatorname{dim} \nu=\frac{h_{M}}{\varepsilon \ell_{M}}
$$

where $h_{M}$ and $\ell_{M}$ denote the asymptotic entropy and the drift of the Brownian motion respectively. Furthermore, $h_{M}=\ell_{M} v$ if and only if $\nu$ is equivalent to the Hausdorff measure of dimension $v / \varepsilon$ on $\left(\partial M, d_{\varepsilon}\right)$.

The first result is folklore and explicitly stated by V. Kaimanovich in the introduction of [24], but we know of no published proof. The second statement is due to F. Ledrappier [33]. Note that more is known: the equality $h_{M}=\ell_{M} v$ is equivalent to the equality of $\nu$ with the canonical conformal measure on $\left(\partial M, d_{\varepsilon}\right)$ for surfaces [34]; and equality of both measures (in any dimension) is possible only if $M$ is a rank 1 symmetric space [6].

\subsection{Fuchsian groups with cusps}

We provide an alternative proof based on the Green metric of a theorem due to Y. Guivarc'h and Y. Le Jan about random walks on Fuchsian groups, see the last corollary of [19].

4 e SÉRIE - TOME $44-2011-$ No $^{\circ}$ 
Theorem 1.10 (Y. Guivarc'h \& Y. Le Jan). - Let $\Gamma$ be a discrete subgroup of $P S L_{2}(\mathbb{R})$ such that the quotient space $\mathbb{H}^{2} / \Gamma$ is not compact but has finite volume. Let $\nu_{1}$ be the harmonic measure on $\mathbb{S}^{1}$ given by a symmetric law $\mu$ with finite support on $\Gamma$. (Almost any trajectory of the random walk converges to a point in $\partial \mathbb{H}^{2}=\mathbb{S}^{1}$ and $\nu_{1}$ is the law of this limit point.) Then $\nu_{1}$ is singular with respect to the Lebesgue measure on $\mathbb{S}^{1}$.

Note that it follows from Theorem 1.5 that $\nu_{1}$ is singular with respect to the Lebesgue measure if and only if its dimension is less than 1.

This theorem was originally derived from results on winding numbers of the geodesic flow, see [19] and [20]. A more recent proof based on ergodic properties of smooth group actions on $\mathbb{S}^{1}$ was obtained by B. Deroin, V. Kleptsyn and A. Navas in [15]. It applies to random walks with a finite first moment.

We shall see how Theorem 1.10 can also be deduced from the hyperbolicity of the Green metric through a rather straightforward argument. We only consider the symmetric and finite support case even though it would also work if the random walk has a first finite moment and if $d_{G} \in \mathscr{D}(\Gamma)$.

We thank B. Deroin, Y. Guivarc'h and Y. Le Jan for enlightening explanations on their theorem.

\subsection{Outline of the paper}

In Section 2, we recall the main facts on hyperbolic groups which will be used in the paper. We introduce the notion of quasiruled structures which enables us to generalize the theory of geodesic hyperbolic metric spaces to a broader setting. In Section 3, we recall the construction of random walks, discuss some of their properties and introduce the Green metric. We also prove Theorem 1.7 and Theorem 1.1. We then draw some consequences on the harmonic measure and the random walk. The following Section 4 deals with the proof of Theorem 1.3. In Section 5, we deal with Theorem 1.5 and its corollary and we conclude with the proof of Theorem 1.10. Finally, Theorem 1.9 is proved in Section 6.

\subsection{Notation}

A distance in a metric space will be denoted either by $d(\cdot, \cdot)$ or $|\cdot-\cdot|$. If $a$ and $b$ are positive,

$a \lesssim b$ means that there is a universal positive constant $u$ such that $a \leq u b$. We will write $a \asymp b$ when both $a \lesssim b$ and $b \lesssim a$ hold. Throughout the article, dependance of a constant on structural parameters of the space will not be notified unless needed. Sometimes, it will be convenient to use Landau's notation $O(\cdot)$.

Acknowledgements. - We thank the anonymous referees for their comments which helped improve the exposition. 


\section{Hyperbolicity in metric spaces}

Let $(X, d)$ be a metric space. It is said to be proper if closed balls of finite radius are compact. A geodesic curve (resp. ray, segment) is a curve isometric to $\mathbb{R}$ (resp. $\mathbb{R}_{+}$, a compact interval of $\mathbb{R}$ ). The space $X$ is said to be geodesic if every pair of points can be joined by a geodesic segment.

Definition. - A metric space $(X, d)$ is $\delta$-hyperbolic $(\delta \geq 0)$ if, for any $w, x, y, z \in X$, the following ultrametric type inequality holds

$$
(y \mid z)_{w} \geq \min \left\{(x \mid y)_{w},(x \mid z)_{w}\right\}-\delta,
$$

where the Gromov inner product is defined as

$$
(x \mid y)_{w} \stackrel{\text { def }}{=}(1 / 2)\{|x-w|+|y-w|-|x-y|\} .
$$

We shall write $(\cdot \mid \cdot)_{w}=(\cdot \mid \cdot)$ when the choice of $w$ is clear from the context.

Given two constants $\lambda \geq 1, c \geq 0$, a map $f: X \rightarrow Y$ between two metric spaces is a $(\lambda, c)$-quasi-isometric embedding if, for any $x, x^{\prime} \in X$, we have

$$
\frac{1}{\lambda}\left|x-x^{\prime}\right|-c \leq\left|f(x)-f\left(x^{\prime}\right)\right| \leq \lambda\left|x-x^{\prime}\right|+c .
$$

The map $f$ is a $(\lambda, c)$-quasi-isometry if, in addition, there exist a quasi-isometric embedding $g: Y \rightarrow X$ and a constant $C$ such that $|g \circ f(x)-x| \leq C$ for any $x \in X$. A quasigeodesic curve (resp. ray, segment) is the image of $\mathbb{R}$ (resp. $\mathbb{R}_{+}$, a compact interval of $\mathbb{R}$ ) by a quasiisometric embedding.

Compactification. - Let $X$ be a proper hyperbolic space, and $w \in X$ a base point. A sequence $\left(x_{n}\right)$ tends to infinity if, by definition, $\left(x_{n} \mid x_{m}\right) \rightarrow \infty$ as $m, n \rightarrow \infty$. The visual or hyperbolic boundary $\partial X$ of $X$ is the set of sequences which tend to infinity modulo the equivalence relation defined by: $\left(x_{n}\right) \sim\left(y_{n}\right)$ if $\left(x_{n} \mid y_{n}\right) \rightarrow \infty$. One may also extend the Gromov inner product to points at infinity in such a way that the inequality

$$
(y \mid z) \geq \min \{(x \mid y),(x \mid z)\}-\delta,
$$

now holds for any points $w, x, y, z \in X \cup \partial X$.

For each $\varepsilon>0$ small enough, there exists a so-called visual metric $d_{\varepsilon}$ on $\partial X$, i.e., which satisfies for any $a, b \in \partial X: d_{\varepsilon}(a, b) \asymp e^{-\varepsilon(a \mid b)}$.

We shall use the notation $B_{\varepsilon}(a, r)$ to denote the ball in the space $\left(\partial X, d_{\varepsilon}\right)$ with center $a$ and radius $r$.

We refer to [17] for the details (chap. 6 and 7).

Busemann functions. - Let us assume that $(X, d)$ is a hyperbolic space. Let $a \in \partial X$, $x, y \in X$. The function

$$
\beta_{a}(x, y) \stackrel{\text { def }}{=} \sup \left\{\limsup _{n \rightarrow \infty}\left[d\left(x, a_{n}\right)-d\left(y, a_{n}\right)\right]\right\},
$$

where the supremum is taken over all sequences $\left(a_{n}\right)_{n}$ in $X$ which tends to $a$, is called the Busemann function at the point $a$.

$4{ }^{\mathrm{e}}$ SÉRIE - TOME $44-2011$ - No 4 
Shadows. - Let $R>0$ and $x \in X$. The shadow $\mho(x, R)$ is the set of points $a \in \partial X$ such that $(a \mid x)_{w} \geq d(w, x)-R$.

Approximating finitely many points by a tree (cf. Theorem 2.4) yields:

Proposition 2.1. - Let $(X, d)$ be a hyperbolic space. For any $\tau \geq 0$, there exist positive constants $C, R_{0}$ such that for any $R>R_{0}, a \in \partial X$ and $x \in X$ such that $(w \mid a)_{x} \leq \tau$,

$$
B_{\varepsilon}\left(a, \frac{1}{C} e^{R \varepsilon} e^{-\varepsilon|w-x|}\right) \subset \mho(x, R) \subset B_{\varepsilon}\left(a, C e^{R \varepsilon} e^{-\varepsilon|w-x|}\right) .
$$

Shadows will enable us to control measures on the boundary of a hyperbolic group, see the lemma of the shadow in the next paragraph.

\subsection{Quasiruled hyperbolic spaces}

For geodesic spaces, hyperbolicity admits many characterizations based on geodesic triangles (cf. Prop. 2.21 from [17]). Most of them still hold when the space $X$ is just a length space (see eg. [42]).

It is known that if $X$ and $Y$ are two quasi-isometric geodesic spaces, then $X$ is hyperbolic if and only if $Y$ is (Theorem 5.12 in [17]). This statement is known to be false in general if we do not assume both spaces to be geodesic (Example 5.12 from [17], and Proposition 2.3 below).

Since quasi-isometries do not preserve small-scales of metric spaces, in particular geodesics, it is therefore important to find other coarse characterizations of hyperbolicity. We propose a setting which enables us to go through the whole theory of quasiconformal measures as if the underlying space was geodesic.

Recall that a metric space is said to be quasigeodesic if there are constants $\lambda, c$ such that any pair of points can be connected by a $(\lambda, c)$-quasigeodesic. The image of a geodesic space by a quasi-isometry is thus quasigeodesic. But as it was mentioned earlier, hyperbolicity need not be preserved.

Definition. - A $\tau$-quasiruler is a quasigeodesic $g: \mathbb{R} \rightarrow X$ (resp. quasisegment $g: I \rightarrow X$, quasiray $g: \mathbb{R}_{+} \rightarrow X$ ) such that, for any $s<t<u$,

$$
(g(s) \mid g(u))_{g(t)} \leq \tau \text {. }
$$

Let $X$ be a metric space. Let $\lambda \geq 1$ and $\tau, c>0$ be constants. A quasiruling structure $\mathscr{G}$ is a set of $\tau$-quasiruled $(\lambda, c)$-quasigeodesics such that any pair of points of $X$ can be joined by an element of $\mathscr{G}$. A metric space will be quasiruled if it admits a quasiruling structure.

TheOREM 2.2. - Let $X$ be a geodesic hyperbolic metric space, and $Y$ a metric space quasi-isometric to $X$. Then $Y$ is hyperbolic if and only if $Y$ is quasiruled.

A quasigeodesic hyperbolic space is quasiruled by [10, Thm 4.1]. The idea of the proof of the converse, which we will not give here, is to approximate the configuration of four points joined by quasirulers by a tree.

In [17], the authors provide an example of a non-hyperbolic metric space quasi-isometric to $\mathbb{R}$. One could wonder if, in the case of groups, the invariance of hyperbolicity holds for quasi-isometric and invariant metrics. This is not the case: 
Proposition 2.3. - For any hyperbolic group, a left-invariant metric quasi-isometric to a word metric exists which is not hyperbolic.

We are grateful to C. Pittet and I. Mineyev for having pointed out to us the metric $d$ in the following proof as a possible candidate.

Proof. - Let $\Gamma$ be a hyperbolic group and let $|$.$| denote a word metric. We define the$ metric

$$
d(x, y)=|x-y|+\log (1+|x-y|) .
$$

Clearly, $|x-y| \leq d(x, y) \leq 2|x-y|$ holds and $d$ is left-invariant by $\Gamma$.

Let us prove that $(\Gamma, d)$ is not quasiruled, hence not hyperbolic by Theorem 2.2.

Let $g$ be a geodesic for $|$.$| which we identify with \mathbb{Z}$. Since $(\Gamma, d)$ is bi-Lipschitz to $(\Gamma,|\cdot|)$, it is a $(2,0)$-quasigeodesic for $d$. But

$$
d(0, n)+d(n, 2 n)-d(0,2 n)=\log \frac{(1+n)^{2}}{1+2 n}
$$

asymptotically behaves as $\log n$. Therefore $g$ is not quasiruled.

Approximate trees. - Approximate trees is an important tool to understand hyperbolicity in geodesic spaces. Here, we state the analogous statement in the setting of hyperbolic quasiruled metric spaces.

TheOREM 2.4. - Let $(X, w)$ be a $\delta$-hyperbolic metric space and let $k \geq 0$.

(i) If $|X| \leq 2^{k}+2$, then there is a finite metric pointed tree $T$ and a map $\phi: X \rightarrow T$ such that:

$\rightarrow \forall x \in X,|\phi(x)-\phi(w)|=|x-w|$,

$\rightarrow \forall x, y \in X,|x-y|-2 k \delta \leq|\phi(x)-\phi(y)| \leq|x-y|$.

(ii) If there are $\tau$-quasiruled rays $\left(X_{i}, w_{i}\right)_{1 \leq i \leq n}$ with $n \leq 2^{k}$ such that $X=\cup X_{i}$, then there is a pointed $\mathbb{R}$-tree $T$ and a map $\phi: X \rightarrow T$ such that

$\rightarrow \forall x \in X,|\phi(x)-\phi(w)|=|x-w|$,

$\rightarrow \forall x, y \in X,|x-y|-2(k+1) \delta-4 c-2 \tau \leq|\phi(x)-\phi(y)| \leq|x-y|$, where $c=\max \left\{\left|w-w_{i}\right|\right\}$.

The proof mimics the proof of the standard case [17, Theorem 2.12].

Visual quasiruling structures. - Let $(X, d, w)$ be a hyperbolic space endowed with a quasiruling structure $\mathscr{G}$. We say that $\mathscr{G}$ is visual if any pair of points in $X \cup \partial X$ can be joined by a $\tau$-quasiruled $(\lambda, c)$-quasigeodesic. If $X$ is a proper space, then any quasiruling structure can be completed into a visual quasiruling structure. Also, if $Y$ is a hyperbolic geodesic proper metric space and $\varphi: Y \rightarrow X$ is a quasi-isometry which maps any geodesic segment to a $\tau$-quasiruler, then the induced quasiruling structure is also visual. This fact can in particular be applied when $Y$ is a locally finite Cayley graph of a non-elementary hyperbolic group $\Gamma,(X, d) \in \mathscr{D}(\Gamma)$ and $\varphi$ is the identity map. Thus one endows $(X, d)$ with a visual quasiruling structure.

Note that, in a hyperbolic space endowed with a visual quasiruling structure, Theorem 2.4 implies that the definition of Busemann functions we gave is equivalent to the classical one 
based on geodesics. One can actually retrieve the metric from the Busemann functions as the next lemma shows.

Lemma 2.5. - Let $(X, w)$ be a pointed hyperbolic quasiruled space with the following quasi-starlike property: there exists $R_{1}$ such that any $x \in X$ is at distance at most $R_{1}$ from a quasiray $[w, a), a \in \partial X$. Then there exists a constant $C$ such that

$$
|| x-y\left|-\sup _{a \in \partial X} \beta_{a}(x, y)\right| \leq C,
$$

for all $x, y \in X$. The constant $C$ depends only on the data $\left(\delta, \lambda, c, \tau, R_{1}\right)$.

Observe that the quasi-starlike property is satisfied as soon as there is a cocompact action of a subgroup of isometries of $X$.

Proof. - From the triangle inequality, we always have $\beta_{a}(x, y) \leq|x-y|$. Now choose $x, y \in X$ and $a \in \partial X$ such that $y$ is at distance at most $R_{1}$ from a quasiray $[w, a)$. If these four points were really sitting on a tree, we would have $\beta_{a}(x, y) \geq|x-y|-R_{1}$. Using approximate trees as in Theorem 2.4, we get the lemma.

Shadows. - Let $(X, d, w)$ be a hyperbolic quasiruled space endowed with a visual quasiruling structure $\mathscr{G}$. We already defined the shadow $\mho(x, R)$ above. An alternative definition is: let $\mho_{\mathscr{G}}(x, R)$ be the set of points $a \in \partial X$ such that there is a quasiruler $[w, a) \in \mathscr{G}$ which intersects

$$
B(x, R)=\{y \in X: d(x, y)<R\} .
$$

The following holds by applying Theorem 2.4, since $\mathscr{G}$ is visual.

Proposition 2.6. - Let $X$ be a hyperbolic space endowed with a visual quasiruling structure $\mathscr{G}$. There exist positive constants $C, R_{0}$ such that for any $R>R_{0}, a \in \partial X$ and $x \in[w, a) \in \mathscr{G}$,

$$
\mho_{\mathscr{G}}(x, R-C) \subset \mho(x, R) \subset \mho_{\mathscr{G}}(x, R+C) .
$$

\subsection{Hyperbolic groups}

Let $X$ be a hyperbolic proper metric space and $\Gamma$ a subgroup of isometries which acts properly discontinuously on $X$, i.e., for any compact sets $K$ and $L$, the number of group elements $\gamma \in \Gamma$ such that $\gamma(K) \cap L \neq \varnothing$ is finite. For any point $x \in X$, its orbit $\Gamma(x)$ accumulates only on the boundary $\partial X$, and its set of accumulation points turns out to be independent of the choice of $x$; by definition, $\overline{\Gamma(x)} \cap \partial X$ is the limit set $\Lambda(\Gamma)$ of $\Gamma$.

An action of a group $\Gamma$ on a metric space is said to be geometric if

1. each element acts by isometry;

2. the action is properly discontinuous;

3. the action is cocompact. 
For example, if $\Gamma$ is a finitely generated group, $S$ is a finite symmetric set of generators, one may consider the Cayley graph $X$ associated with $S$ : the set of vertices are the elements of the group, and pairs $\left(\gamma, \gamma^{\prime}\right) \in \Gamma \times \Gamma$ define an edge if $\gamma^{-1} \gamma^{\prime} \in S$. Endowing $X$ with the metric which makes each edge isometric to the segment $[0,1]$ defines the word metric associated with $S$. It turns $X$ into a geodesic proper metric space on which $\Gamma$ acts geometrically by left-translation.

A group $\Gamma$ is hyperbolic if it acts geometrically on a geodesic proper hyperbolic metric space (e.g. a locally finite Cayley graph). In this case, one has $\Lambda(\Gamma)=\partial X$.

We will say that a metric space $(X, d)$ is quasi-isometric to the group $\Gamma$ if it is quasiisometric to a locally finite Cayley graph of $\Gamma$.

Let $\Gamma$ be a hyperbolic group geometrically acting on $(X, d)$. The action of $\Gamma$ extends to the boundary. Busemann functions, visual metrics and the action of $\Gamma$ are related by the following property: for any $a \in \partial X$ and any $\gamma \in \Gamma$, there exists a neighborhood $V$ of $a$ such that, for any $b, c \in V$,

$$
d_{\varepsilon}(\gamma(b), \gamma(c)) \asymp L_{\gamma}(a) d_{\varepsilon}(b, c)
$$

where $L_{\gamma}(a) \stackrel{\text { def }}{=} e^{\varepsilon \beta_{a}\left(w, \gamma^{-1}(w)\right)}$. Moreover, $\Gamma$ also acts on measures on $\partial X$ through the rule $\gamma^{*} \rho(A) \stackrel{\text { def }}{=} \rho(\gamma A)$. In other words, $\gamma^{*} \rho$ is the pull-back of $\rho$ by $\gamma$ (this is particularly convenient for defining quasiconformal measures, see (ii) of Theorem 2.7).

\subsection{Quasiconformal measures}

The next theorem summarizes the main properties of quasiconformal measures on the boundary of $X$. It was proved by M. Coornaert in [14] in the context of geodesic spaces. We state here a more general version to cover the case $d \in \mathscr{D}(\Gamma)$.

Theorem 2.7. - Let $(X, d)$ be a proper quasiruled hyperbolic space endowed with a geometric action of a non-elementary hyperbolic group $\Gamma$. For any small enough $\varepsilon>0$, we have $0<\operatorname{dim}_{H}\left(\partial X, d_{\varepsilon}\right)<\infty$ and

$$
v \stackrel{\text { def }}{=} \lim \sup \frac{1}{R} \log |\{\Gamma(w) \cap B(w, R)\}|=\varepsilon \cdot \operatorname{dim}_{H}\left(\partial X, d_{\varepsilon}\right) .
$$

Let $\rho$ be the Hausdorff measure on $\partial X$ of dimension $\alpha \stackrel{\text { def }}{=} v / \varepsilon$;

(i) $\rho$ is Ahlfors-regular of dimension $\alpha$, i.e., for any $a \in \partial X$, for any $r \in(0$, diam $\partial X)$, $\rho\left(B_{\varepsilon}(a, r)\right) \asymp r^{\alpha}$. In particular, $0<\rho(\partial X)<\infty$.

(ii) $\rho$ is a quasiconformal measure i.e., for any isometry $\gamma$ we have $\rho \ll \gamma^{*} \rho \ll \rho$ and

$$
\frac{d \gamma^{*} \rho}{d \rho} \asymp\left(L_{\gamma}\right)^{\alpha} \rho \text { a.e. }
$$

(iii) The action of $\Gamma$ is ergodic for $\rho$ i.e., for any $\Gamma$-invariant Borelian $B$ of $\partial X$,

$$
\rho(B)=0 \text { or } \rho(\partial X \backslash B)=0 .
$$

Moreover, if $\rho^{\prime}$ is another $\Gamma$-quasiconformal measure, then $\rho \ll \rho^{\prime} \ll \rho$ and $\frac{d \rho}{d \rho^{\prime}} \asymp 1$ a.e. and

$$
|\{\Gamma(w) \cap B(w, R)\}| \asymp e^{v R}
$$


The whole theory of quasiconformal measures for hyperbolic groups acting on geodesic spaces in [14] is based on the existence of approximate trees. Therefore, the same proof as in [14] leads to Theorem 2.7.

The class of measures thus defined on $\partial X$ is called the Patterson-Sullivan class. It does not depend on the choice of the parameter $\varepsilon$ but it does depend on the metric $d$.

The study of quasiconformal measures yields the following key estimate [14]:

Lemma 2.8 (Lemma of the shadow). - Under the assumptions of Theorem 2.7, there exists $R_{0}$, such that if $R>R_{0}$, then, for any $x \in X$,

$$
\rho(\mho(x, R)) \asymp e^{-v d(w, x)}
$$

where the implicit constants do not depend on $x$.

\section{Random walks and Green metrics for hyperbolic groups}

Let $\Gamma$ be a non-elementary hyperbolic group. We fix a metric $(X, d) \in \mathscr{D}(\Gamma)$ with a base point $w \in X$, and we consider a symmetric probability measure $\mu$ on $\Gamma$ with finite first moment the support of which generates $\Gamma$.

Let $\left(Z_{n}\right)_{n}$ be the random walk starting from the neutral element $e$ associated with $\mu$. Thus, for each $n, Z_{n}$ is a random variable taking its values in $\Gamma$. We use the notation $Z_{n}(w)$ for the image of the base point $w \in X$ by $Z_{n}$.

Since $\Gamma$ is assumed to be non-elementary, $\Gamma$ is non-amenable so not only is the random walk always transient, the rate of escape

$$
\ell \stackrel{\text { def }}{=} \lim _{n} \frac{d\left(w, Z_{n}(w)\right)}{n}
$$

is also positive (cf. [28, §7.3]).

There are different ways to prove that almost any trajectory of the random walk has a limit point $Z_{\infty}(w) \in \partial X$. We recall below a theorem by V. Kaimanovich (cf. Theorem 7.3 in [28] and $\$ 7.4$ therein) since it contains some information on the way $\left(Z_{n}(w)\right)$ actually tends to $Z_{\infty}(w)$ that will be used later.

THeorem 3.1 (V. Kaimanovich). - Let $\Gamma$ be a non-elementary hyperbolic group and $(X, d) \in \mathscr{D}(\Gamma)$, and let us consider a symmetric probability measure $\mu$ with finite first moment the support of which generates $\Gamma$. Then $\left(Z_{n}(w)\right)$ almost surely converges to a point $Z_{\infty}(w)$ on the boundary.

For any $a \in \partial X$, we choose a quasigeodesic $[w, a)$ from $w$ to a in a measurable way.

For any $n$, there is a measurable map $\pi_{n}$ from $\partial X$ to $X$ such that $\pi_{n}(a) \in[w, a)$, and, for almost any trajectory of the random walk,

$$
\lim _{n \rightarrow \infty} \frac{\left|Z_{n}(w)-\pi_{n}\left(Z_{\infty}(w)\right)\right|}{n}=0 .
$$


The actual result was proved for geodesic metrics $d$. Once proved in a locally finite Cayley graph, one may then use a quasi-isometry to get the statement in this generality.

The estimate (2) will be improved in Corollary 3.9 under the condition that $d_{G}$ belongs to $\mathscr{D}(\Gamma)$.

The harmonic measure $\nu$ is then the law of $Z_{\infty}(w)$, i.e., it is the probability measure on $\partial X$ such that $\nu(A)$ is the probability that $Z_{\infty}(w)$ belongs to the set $A$. More generally, we let $\nu_{\gamma}$ be the harmonic measure for the random walk started at the point $\gamma(w), \gamma \in \Gamma$, i.e., the law of $\gamma\left(Z_{\infty}(w)\right)$. Comparing with the action of $\Gamma$ on $\partial X$, we see that $\gamma^{*} \nu=\nu_{\gamma^{-1}}$.

\subsection{Properness of the Green metric}

Let $\Gamma$ be a countable group and $\mu$ a symmetric law the support of which generates $\Gamma$. We endow $\Gamma$ with the Green metric associated to $\mu$.

Lemma 3.2. - Let $\mu$ be a symmetric probability measure on $\Gamma$ which defines a transient random walk. Then $\left(\Gamma, d_{G}\right)$ is a proper metric space, i.e., balls of finite radius are finite.

Proof. - It is enough to prove that $G(e, x)$ tends to 0 as $x$ leaves any finite set.

Fix $n \geq 1$; by definition of convolution and by the Cauchy-Schwarz inequality,

$$
\mu^{2 n}(x)=\sum_{y \in \Gamma} \mu^{n}(y) \mu^{n}\left(y^{-1} x\right) \leq \sqrt{\sum_{y \in \Gamma} \mu^{n}(y)^{2}} \sqrt{\sum_{y \in \Gamma} \mu^{n}\left(y^{-1} x\right)^{2}} .
$$

Since we are summing over the same set, it follows that

$$
\sum_{y \in \Gamma} \mu^{n}(y)^{2}=\sum_{y \in \Gamma} \mu^{n}\left(y^{-1} x\right)^{2}
$$

and the symmetry of $\mu$ implies that

$$
\sum_{y \in \Gamma} \mu^{n}(y)^{2}=\sum_{y \in \Gamma} \mu^{n}(y) \mu^{n}\left(y^{-1}\right)=\mu^{2 n}(e) .
$$

Therefore, $\mu^{2 n}(x) \leq \mu^{2 n}(e)$. Similarly,

$$
\mu^{2 n+1}(x)=\sum_{y \in \Gamma} \mu(y) \mu^{2 n}\left(y^{-1} x\right) \leq \sum_{y \in \Gamma} \mu(y) \mu^{2 n}(e) \leq \mu^{2 n}(e) .
$$

Since the walk is transient, it follows that $G(e, e)$ is finite, so, given $\varepsilon>0$, there is some $k \geq 1$ such that

$$
\sum_{n \geq k} \mu^{2 n}(e) \leq \sum_{n \geq 2 k} \mu^{n}(e) \leq \varepsilon .
$$

On the other hand, since $\mu^{n}$ is a probability measure for all $n$, there is some finite subset $K$ of $\Gamma$ such that, for all $n \in\{0, \ldots, 2 k-1\}, \mu^{n}(K) \geq 1-\varepsilon /(2 k)$. Therefore, if $x \notin K$, then

$$
G(e, x)=\sum_{0 \leq n<2 k} \mu^{n}(x)+\sum_{n \geq 2 k} \mu^{n}(x) \leq \sum_{0 \leq n<2 k} \mu^{n}(\Gamma \backslash K)+2 \sum_{n \geq k} \mu^{2 n}(e) \leq \varepsilon+2 \varepsilon .
$$

The lemma follows. 


\subsection{The Martin boundary}

In this paragraph, we prove Theorem 1.7. The key steps are similar to Ancona's original approach, but phrased in a more geometrical language (note that there is a mistake in the proof of [26, Thm 3.1]).

Let $\Gamma$ be a countable group and $\mu$ be a symmetric probability measure on $\Gamma$. We assume that the support of $\mu$ generates $\Gamma$ and that the corresponding random walk is transient.

A non-negative function $h$ on $\Gamma$ is $\mu$-harmonic (harmonic for short) if, for all $x \in \Gamma$,

$$
h(x)=\sum_{y \in \Gamma} h(y) \mu\left(x^{-1} y\right) .
$$

A positive harmonic function $h$ is minimal if any other positive harmonic function $v$ smaller than $h$ is proportional to $h$.

Let us briefly recall the construction of the Martin boundary $\partial_{M} \Gamma$ : let $\Psi: \Gamma \rightarrow C(\Gamma)$ be defined by $y \longmapsto K_{y}=K(\cdot, y)$. Here $C(\Gamma)$ is the space of real-valued functions defined on $\Gamma$ endowed with the topology of pointwise convergence. It turns out that $\Psi$ is injective and thus we may identify $\Gamma$ with its image. The closure of $\Psi(\Gamma)$ is compact in $C(\Gamma)$ and, by definition, $\partial_{M} \Gamma=\overline{\Psi(\Gamma)} \backslash \Psi(\Gamma)$ is the Martin boundary. In the compact space $\Gamma \cup \partial_{M} \Gamma$, for any initial point $x$, the random walk $Z_{n}(x)$ almost surely converges to some random variable $Z_{\infty}(x) \in \partial_{M} \Gamma$ (see for instance E. Dynkin [16], A. Ancona [2] or W. Woess [44]).

To every point $\xi \in \partial_{M} \Gamma$ corresponds a positive harmonic function $K_{\xi}$. Every minimal function arises in this way: if $h$ is minimal, then there are a constant $c>0$ and $\xi \in \partial_{M} \Gamma$ such that $h=c K_{\xi}$. We denote by $\partial_{m} \Gamma$ the subset of $\partial_{M} \Gamma$ consisting of (normalised) minimal positive harmonic functions.

Choquet's integral representation implies that, for any positive harmonic function $h$, there is a unique probability measure $\kappa^{h}$ on $\partial_{m} \Gamma$ such that

$$
h=\int K_{\xi} d \kappa^{h}(\xi) \text {. }
$$

We will also use L. Naïm's kernel $\Theta$ on $\Gamma \times \Gamma$ defined by

$$
\Theta(x, y) \stackrel{\text { def }}{=} \frac{G(x, y)}{G(e, x) G(e, y)}=\frac{K_{y}(x)}{G(e, x)} .
$$

As the Martin kernel, Naïm's kernel admits a continuous extension to $\Gamma \times\left(\Gamma \cup \partial_{M} \Gamma\right)$. In terms of the Green metric, one gets

$$
\log \Theta(x, y)=2(x \mid y)_{e}^{G}-\log G(e, e),
$$

where $(x \mid y)_{e}^{G}$ denotes the Gromov product with respect to the Green metric. See [39] for properties of this kernel.

We shall from now on assume that the Green metric $d_{G}$ is hyperbolic. Then it has a visual boundary that we denote by $\partial_{G} \Gamma$. We may also compute the Busemann function in the metric $d_{G}$, say $\beta_{a}^{G}$. Sending $y$ to some point $a \in \partial_{G} \Gamma$ in the equation $d_{G}(e, y)-d_{G}(x, y)=$ $\log K(x, y)$, we get that $\beta_{a}^{G}(e, x)=\log K_{a}(x)$. 
We now start preparing the proof of Theorem 1.7 in the next lemma and proposition. We define an equivalence relation $\sim_{M}$ on $\partial_{M} \Gamma$ : say that $\xi \sim_{M} \zeta$ if there exists a constant $C \geq 1$ such that

$$
\frac{1}{C} \leq \frac{K_{\xi}}{K_{\zeta}} \leq C .
$$

Given $\xi \in \partial_{M} \Gamma$, we denote by $M(\xi)$ the class of $\xi$.

We first derive some properties of this equivalence relation:

Lemma 3.3. - (i) There exists a constant $E \geq 1$ such that for all sequences $\left(x_{n}\right)$ and $\left(y_{n}\right)$ in $\Gamma$ converging to $\xi$ and $\zeta$ in $\partial_{M} \Gamma$ respectively and such that $\Theta\left(x_{n}, y_{n}\right)$ tends to infinity, then

$$
\frac{1}{E} \leq \frac{K_{\xi}}{K_{\zeta}} \leq E
$$

in particular, $\xi \sim_{M} \zeta$.

(ii) For any $\xi \in \partial_{M} \Gamma$, there is some $\zeta \in M(\xi)$ and a sequence $\left(y_{n}\right)$ in $\Gamma$ which tends to some point $a \in \partial_{G} \Gamma$ in the sense of Gromov, to $\zeta \in \partial_{M} \Gamma$ in the sense of Martin and such that $\Theta\left(y_{n}, \xi\right)$ tends to infinity.

(iii) Let $\xi, \zeta \in \partial_{M} \Gamma$. If $\zeta \notin M(\xi)$, then there is a neighborhood $V(\zeta)$ of $\zeta$ in $\Gamma$ and a constant $M$ such that

$$
K_{\xi}(x) \leq M G(e, x)
$$

for any $x \in V(\zeta)$.

Proof. - (i) Fix $z \in \Gamma$ and $n$ large enough so that $\left(x_{n} \mid y_{n}\right)_{e}^{G} \gg d_{G}(e, z)$; we consider the approximate tree $T$ associated with $F=\left\{e, z, x_{n}, y_{n}\right\}$ and the $(1, C)$-quasi-isometry $\varphi:\left(F, d_{G}\right) \rightarrow\left(T, d_{T}\right)$ (cf. Theorem 2.4).

On the tree $T$,we have

$$
\left|d_{T}\left(\varphi(e), \varphi\left(x_{n}\right)\right)-d_{T}\left(\varphi(z), \varphi\left(x_{n}\right)\right)\right|=\left|d_{T}\left(\varphi(e), \varphi\left(y_{n}\right)\right)-d_{T}\left(\varphi(z), \varphi\left(y_{n}\right)\right)\right|,
$$

so that

$$
\left|\left(d_{G}\left(e, x_{n}\right)-d_{G}\left(z, x_{n}\right)\right)-\left(d_{G}\left(e, y_{n}\right)-d_{G}\left(z, y_{n}\right)\right)\right| \leq 2 C .
$$

In terms of the Martin kernel,

$$
\left|\log K_{x_{n}}(z)-\log K_{y_{n}}(z)\right| \leq 2 C .
$$

Letting $n$ go to infinity yields the result.

(ii) Let $\left(y_{n}\right)$ be a sequence such that

$$
\lim K_{\xi}\left(y_{n}\right)=\sup K_{\xi} .
$$

Since $K_{\xi}$ is harmonic, the maximum principle implies that $\left(y_{n}\right)$ leaves any compact set. But the walk is symmetric and transient so Lemma 3.2 implies that $G\left(e, y_{n}\right)$ tends to 0 .

Furthermore, for $n$ large enough, $K_{\xi}\left(y_{n}\right) \geq K_{\xi}(e)=1$, so that

$$
\Theta\left(y_{n}, \xi\right) \geq \frac{1}{G\left(e, y_{n}\right)} \rightarrow \infty .
$$

Let $\left(x_{n}\right)$ be a sequence in $\Gamma$ which tends to $\xi$. For any $n$, there is some $m$ such that

$$
\left|K_{\xi}\left(y_{n}\right)-K_{x_{m}}\left(y_{n}\right)\right| \leq G\left(e, y_{n}\right) .
$$

4 e SÉRIE - TOME $44-2011$ - No 4 
It follows that

$$
\Theta\left(y_{n}, x_{m}\right) \geq \Theta\left(y_{n}, \xi\right)-\frac{\left|K_{\xi}\left(y_{n}\right)-K_{x_{m}}\left(y_{n}\right)\right|}{G\left(e, y_{n}\right)} \geq \Theta\left(y_{n}, \xi\right)-1
$$

Therefore, applying part (i) of the lemma, we see that any limit point of $\left(y_{n}\right)$ in $\partial_{M} \Gamma$ belongs to $M(\xi)$.

Moreover, for any such limit point $\zeta \in \partial_{M} \Gamma$, we get that

$$
\Theta\left(y_{n}, \zeta\right) \geq \frac{1}{E} \Theta\left(y_{n}, \xi\right)
$$

Applying the same argument as above, we see that, for any $M>0$, there is some $n$ and $m_{n}$ such that, if $m \geq m_{n}$ then

$$
\Theta\left(y_{n}, y_{m}\right) \geq M-1 \text {. }
$$

From (3) we conclude, using a diagonal procedure, that there exist a subsequence $\left(n_{k}\right)$ such that $\left(y_{n_{k}}\right)$ tends to infinity in the Gromov topology.

(iii) Since $\zeta \notin M(\xi)$, there is a neighborhood $V(\zeta)$ and a constant $M$ such that $\Theta(x, \xi) \leq M$ for all $x \in V(\zeta)$. Otherwise, we would find $y_{n} \rightarrow \zeta$ with $\Theta\left(y_{n}, \xi\right)$ going to infinity, and the argument above would imply $\zeta \in M(\xi)$. Therefore,

$$
K_{\xi}(x) \leq M G(e, x) .
$$

Proposition 3.4. - Every Martin point is minimal.

Proof. - We observe that if $K_{\xi}$ is minimal, then $M(\xi)=\{\xi\}$. Indeed, if $\zeta \in M(\xi)$, then

$$
K_{\xi} \geq K_{\xi}-\frac{1}{C} K_{\zeta} \geq 0
$$

for some constant $C \geq 1$. The minimality of $K_{\xi}$ implies that $K_{\xi}$ and $K_{\zeta}$ are proportional and, since their value at $e$ is $1, K_{\xi}=K_{\zeta}$, i.e., $\xi=\zeta$.

Let $\xi \in \partial_{M} \Gamma$. There is a unique probability measure $\kappa^{\xi}$ on $\partial_{m} \Gamma$ such that

$$
K_{\xi}=\int K_{\zeta} d \kappa^{\xi}(\zeta)
$$

By Fatou-Doob-Naïm Theorem, for $\kappa^{\xi}$-almost every $\zeta$, the ratio $G(e, x) / K_{\xi}(x)$ tends to 0 as $x$ tends to $\zeta$ in the fine topology [2, Thm. II.1.8]. From Lemma 3.3 (iii), it follows that $\kappa^{\xi}$ is supported by $M(\xi)$. In particular, $M(\xi)$ contains a minimal point.

Proof of Theorem 1.7. - Since every Martin point is minimal, Lemma 3.3 (ii), implies that for every $\xi \in \partial_{M} \Gamma$, there is some sequence $\left(x_{n}\right)$ in $\Gamma$ which tends to $\xi$ in the Martin topology and to some point $a$ in the hyperbolic boundary as well.

Let us prove that the point $a$ does not depend on the sequence. If $\left(y_{n}\right)$ is another sequence tending to $\xi$, then

$$
\limsup _{n, m \rightarrow \infty} \Theta\left(x_{n}, y_{m}\right)=\infty
$$

because $\Theta\left(\xi, x_{n}\right)$ tends to infinity. Therefore, there is a subsequence of $\left(y_{n}\right)$ which tends to $a$ in the Gromov topology. Since we have only one accumulation point, it follows that $a$ is welldefined. This defines a map $\phi: \partial_{M} \Gamma \rightarrow \partial_{G} \Gamma$.

Now, if $\left(x_{n}\right)$ tends to $a$ in the Gromov topology, then it has only one accumulation point in the Martin boundary as well by Lemma 3.3 (i). So the map $\phi$ is injective. The surjectivity follows from the compactness of $\partial_{M} \Gamma$. 
To conclude the proof, it is enough to prove the continuity of $\phi$ since $\partial_{M} \Gamma$ is compact. Let $M>0$ and $\xi \in \partial_{M} \Gamma$ be given. We consider a sequence $\left(x_{n}\right)$ which tends to $\xi$ as in Lemma 3.3. Let $C$ be the constant given by Theorem 2.4 for 4 points. We pick $n$ large enough so that $\left(x_{n} \mid \phi(\xi)\right)_{e}^{G} \geq M+2 C+\log 2$. Let

$$
A=\min \left\{K_{\xi}(x), x \in B_{G}\left(e, d_{G}\left(x_{n}, e\right)\right)\right\} .
$$

Let $\zeta \in \partial_{M} \Gamma$ such that $\left|K_{\xi}-K_{\zeta}\right| \leq(A / 2)$ on $B_{G}\left(e, d_{G}\left(x_{n}, e\right)\right)$. It follows that

$$
1 / 2 \leq \frac{K_{\zeta}}{K_{\xi}} \leq 3 / 2
$$

Approximating $\left\{e, x_{n}, \phi(\xi), \phi(\zeta)\right\}$ by a tree, we conclude that $(\phi(\xi) \mid \phi(\zeta))_{e}^{G} \geq M$, proving the continuity of $\phi$.

\subsection{Hyperbolicity of the Green metric}

We start with a characterization of the hyperbolicity of the Green metric in the quasiisometry class of the group.

Proposition 3.5. - Let $\Gamma$ be a non-elementary hyperbolic group and $\mu$ a symmetric probability measure with Green function $G$. We fix a finite generating set $S$ and consider the associated word metric $d_{w}$. The Green metric $d_{G}$ is quasi-isometric to $d_{w}$ and hyperbolic if and only if the following two conditions are satisfied.

(ED) There are positive constants $C_{1}$ and $c_{1}$ such that, for all $\gamma \in \Gamma$,

$$
G(e, \gamma) \leq C_{1} e^{-c_{1} d_{w}(e, \gamma)} .
$$

(QR) For any $r \geq 0$, there exists a positive constant $C(r)$ such that

$$
G(e, \gamma) \leq C(r) G\left(e, \gamma^{\prime}\right) G\left(\gamma^{\prime}, \gamma\right)
$$

whenever $\gamma, \gamma^{\prime} \in \Gamma$ and $\gamma^{\prime}$ is at distance at most $r$ from a $d_{w}$-geodesic segment between $e$ and $\gamma$.

Proof. - We first assume that $d_{G} \in \mathscr{D}(\Gamma)$. The quasi-isometry property implies that condition (ED) holds. The second condition (QR) follows from Theorem 2.2.

Indeed, since $d_{G}$ is hyperbolic and quasi-isometric to a word distance, then $\left(\Gamma, d_{G}\right)$ is quasiruled. This is sufficient to ensure that condition (QR) holds for $r=0$. The general case $r \geq 0$ follows: let $y$ be the closest point to $\gamma^{\prime}$ on a geodesic between $e$ and $\gamma$ and note that

$d_{G}\left(e, \gamma^{\prime}\right)+d_{G}\left(\gamma^{\prime}, \gamma\right) \leq d_{G}(e, y)+d_{G}(y, \gamma)+2 d_{G}\left(y, \gamma^{\prime}\right) \leq \log C(0)+d_{G}(e, \gamma)+2 d_{G}\left(y, \gamma^{\prime}\right)$.

Thus one may choose $C(r)=C(0) \exp (2 c)$ where $c=\sup d_{G}\left(y, \gamma^{\prime}\right)$ for all pair $y, \gamma^{\prime}$ at distance less than $r$. This last sup is finite because $d_{G}$ is quasi-isometric to a word metric.

For the converse, we assume that both conditions (ED) and (QR) hold and let $C=\max \left\{d_{G}(e, s), s \in S\right\}$. For any $\gamma \in \Gamma$, we consider a $d_{w}$-geodesic $\left\{\gamma_{j}\right\}$ joining $e$ to $\gamma$. It follows that

$$
d_{G}(e, \gamma) \leq \sum_{j} d_{G}\left(\gamma_{j}, \gamma_{j+1}\right) \leq C d_{w}(e, \gamma)
$$

From (ED), we obtain

$$
d_{G}(e, \gamma) \geq c_{1} d_{w}(e, \gamma)-\log C_{1}
$$

$4^{\text {e }}$ SÉRIE - TOME $44-2011-$ No $^{\circ}$ 
Since both metrics are left-invariant, it follows that $d_{w}$ and $d_{G}$ are quasi-isometric.

Condition (QR) implies that $d_{w}$-geodesics are not only quasigeodesics for $d_{G}$, but also quasirulers. Indeed, since the two functions $F$ and $G$ only differ by a multiplicative factor, condition (QR) implies that there is a constant $\tau$ such that, for any $d_{w}$-geodesic segment $\left[\gamma_{1}, \gamma_{2}\right]$ and any $\gamma \in\left[\gamma_{1}, \gamma_{2}\right]$, we have

$$
d_{G}\left(\gamma_{1}, \gamma\right)+d_{G}\left(\gamma, \gamma_{2}\right) \leq 2 \tau+d_{G}\left(\gamma_{1}, \gamma_{2}\right)
$$

Theorem 2.2 (iii) implies (i), implies that $\left(\Gamma, d_{G}\right)$ is a hyperbolic space.

To prove the first statement of Theorem 1.1, it is now enough to establish the following lemma.

Lemma 3.6. - Let $\Gamma$ be a non-elementary hyperbolic group, and $\mu$ a symmetric probability measure with finite exponential moment the support of which generates $\Gamma$. Then condition (ED) holds.

When $\mu$ is finitely supported, the lemma was proved by A. Ancona using potential theory, see [1, pp. 5 and 10] and [2, p. 92], and by S. Blachère and S. Brofferio using the CarneVaropoulos estimate [8].

Proof. - Let us fix a word metric $d_{w}$ induced by a finite generating set $S$, so that $d_{w} \in \mathscr{D}(\Gamma)$.

Since $\Gamma$ is non-amenable, Kesten's criterion implies that there are positive constants $C$ and $a$ such that

$$
\forall \gamma \in \Gamma, \quad \mu^{n}(\gamma) \leq C e^{-a n}
$$

For a proof, see [44, Cor. 12.5].

We assume that $\mathbb{E}\left[\exp \lambda d_{w}\left(e, Z_{1}\right)\right]=E<\infty$ for a given $\lambda>0$. For any $b>0$, it follows from the exponential Tchebychev inequality that

$$
\mathbb{P}\left[\sup _{1 \leq k \leq n} d_{w}\left(e, Z_{k}\right) \geq n b\right] \leq e^{-\lambda b n} \mathbb{E}\left[\exp \left(\lambda \sup _{1 \leq k \leq n} d_{w}\left(e, Z_{k}\right)\right)\right]
$$

But then, for $k \leq n$,

$$
d_{w}\left(e, Z_{k}\right) \leq \sum_{1 \leq j \leq n-1} d_{w}\left(Z_{j}, Z_{j+1}\right)=\sum_{1 \leq j \leq n-1} d_{w}\left(e, Z_{j}^{-1} Z_{j+1}\right) .
$$

The increments $\left(Z_{j}^{-1} Z_{j+1}\right)$ are independent random variables and all follow the same law as $Z_{1}$. Therefore

$$
\mathbb{P}\left[\sup _{1 \leq k \leq n} d_{w}\left(e, Z_{k}\right) \geq n b\right] \leq e^{-\lambda b n} E^{n}=e^{(-\lambda b+\log E) n} .
$$

We choose $b$ large enough so that $c \stackrel{\text { def }}{=}-\lambda b+\log E<0$.

We have

$$
G(e, \gamma)=\sum_{n} \mu^{n}(\gamma)=\sum_{1 \leq k \leq|\gamma| / b} \mu^{k}(\gamma)+\sum_{k>|\gamma| / b} \mu^{k}(\gamma)
$$


where we have set $|\gamma|=d_{w}(e, \gamma)$. The estimates (5) and (4) respectively imply that

$$
\begin{aligned}
\sum_{1 \leq k \leq|\gamma| / b} \mu^{k}(\gamma) & \leq \frac{|\gamma|}{b} \sup _{1 \leq k \leq|\gamma| / b} \mu^{k}(\gamma) \leq \frac{|\gamma|}{b} \mathbb{P}\left[\exists k \leq|\gamma| / b \text { s.t. } Z_{k}=\gamma\right] \\
& \leq \frac{|\gamma|}{b} \mathbb{P}\left[\sup _{1 \leq k \leq|\gamma| / b} d_{w}\left(e, Z_{k}\right) \geq|\gamma|\right] \lesssim|\gamma| e^{-c|\gamma|}
\end{aligned}
$$

and

$$
\sum_{k>|\gamma| / b} \mu^{k}(\gamma) \lesssim e^{-(a / b)|\gamma|}
$$

Therefore, (ED) holds.

When $\Gamma$ is hyperbolic and $\mu$ has finite support, A. Ancona [2] proved that the Martin boundary is homeomorphic to the visual boundary $\partial X$. The key point in his proof is the following estimate (see [44, Thm. 27.12] and Theorem 1.7).

Theorem 3.7 (A. Ancona). - Let $\Gamma$ be a non-elementary hyperbolic group, $X$ a locally finite Cayley graph endowed with a geodesic metric $d$ so that $\Gamma$ acts canonically by isometries, and let $\mu$ be a finitely supported symmetric probability measure the support of which generates $\Gamma$. For any $r \geq 0$, there is a constant $C(r) \geq 1$ such that

$$
F(x, v) F(v, y) \leq F(x, y) \leq C(r) F(x, v) F(v, y)
$$

whenever $x, y \in X$ and $v$ is at distance at most $r$ from a geodesic segment between $x$ and $y$.

This implies together with Lemma 3.6 that when $\mu$ is finitely supported, both conditions (ED) and (QR) hold. Therefore, Proposition 3.5 implies that $d_{G} \in \mathscr{D}(\Gamma)$. We have just established the first statement of Corollary 1.2.

\subsection{Martin kernel vs Busemann function: end of the proof of Theorem 1.1}

We assume that $X=\Gamma$ equipped with the Green metric $d_{G}$ belongs to $\mathscr{D}(\Gamma)$ throughout this paragraph.

Notation. - When we consider notions with respect to $d_{G}$, we will add the exponent $G$ to distinguish them from the same notions in the initial metric $d$. Thus Busemann functions for $d_{G}$ will be written $\beta_{a}^{G}$. The visual metric on $\partial X$ seen from $w$ for the original metric will be denoted by $d_{\varepsilon}$, and by $d_{\varepsilon}^{G}$ for the one coming from $d_{G}$. Balls at infinity will be denoted by $B_{\varepsilon}$ and $B_{\varepsilon}^{G}$.

Let us recall that the Martin kernel is defined by

$$
K(x, y)=\frac{F(x, y)}{F(w, y)}=\exp \left\{d_{G}(w, y)-d_{G}(x, y)\right\} .
$$

By definition of the Martin boundary $\partial_{M} X$, the kernel $K(x, y)$ continuously extends to a $\mu$-harmonic positive function $K_{a}(\cdot)$ when $y$ tends to a point $a \in \partial_{M} X$. We recall that, by Theorem 1.7, we may — and will—identify $\partial_{M} X$ with the visual boundary $\partial X$.

4 e SÉRIE - TOME $44-2011-$ No $^{\text {o }}$ 
As we already mentioned $\Gamma$ acts on $\partial_{M} X$, so on its harmonic measure and we have $\gamma^{*} \nu=\nu_{\gamma^{-1}}$. Besides, see e.g. G. Hunt [22] or W. Woess [44, Th. 24.10] for what follows, $\nu$ and $\nu_{\gamma}$ are absolutely continuous and their Radon-Nikodym derivatives satisfy

$$
\frac{d \nu_{\gamma}}{d \nu}(a)=K_{a}(\gamma(w))
$$

We already computed the Busemann function in the metric $d_{G}$ in part 3.2: $\beta_{a}^{G}(w, x)=\log K_{a}(x)$. Thus we have proved that

$$
\frac{d \gamma^{*} \nu}{d \nu}(a)=\exp \beta_{a}^{G}\left(w, \gamma^{-1} w\right)
$$

It follows at once that $\nu$ is a quasiconformal measure on $\left(\partial X, d_{\varepsilon}^{G}\right)$ of dimension $1 / \varepsilon$. Actually, $\nu$ is even a conformal measure since we have a genuine equality above. Therefore $\nu$ belongs to the Patterson-Sullivan class associated with the metric $d_{G}$. According to Theorem 2.7 , it is in particular comparable to the Hausdorff measure for the corresponding visual metric. This ends both the proofs of Theorem 1.1 and of Corollary 1.2.

We note that, comparing the statements in Theorem 1.1 (ii) and Theorem 2.7, we recover the equality $v_{G}=1$ already noticed in [8] for random walks on non-amenable groups. See also [9].

\subsection{Consequences}

We now draw consequences of the hyperbolicity of the Green metric.

3.5.1. Deviation inequalities. - We study the lateral deviation of the position of the random walk with respect to the quasiruler $\left[w, Z_{\infty}(w)\right)$ where, for any $x \in X$ and $a \in \partial X$, we chose an arbitrary quasiruler $[x, a)$ from $x$ to $a$ in a measurable way.

Proposition 3.8. - Assume that $\Gamma$ is a non-elementary hyperbolic group, $(X, d) \in \mathscr{D}(\Gamma)$, and $\mu$ is a symmetric law so that the associated Green metric belongs to $\mathscr{D}(\Gamma)$. The following holds

(i) There is a positive constant $b$ so that, for any $D \geq 0$ and $n \geq 0$,

$$
\mathbb{P}\left[d\left(Z_{n}(w),\left[w, Z_{\infty}(w)\right)\right) \geq D\right] \lesssim e^{-b D} .
$$

(ii) There is a constant $\tau_{0}$ such that for any positive integers $m, n, k$,

$$
\mathbb{E}\left[\left(Z_{m}(w) \mid Z_{m+n+k}(w)\right)_{Z_{m+n}(w)}\right] \leq \tau_{0} .
$$

Proof of $(i)$. - Observe that

$$
\begin{aligned}
\mathbb{P}\left[d\left(Z_{n}(w),\left[w, Z_{\infty}(w)\right)\right) \geq D\right] & =\sum_{\gamma \in \Gamma} \mathbb{P}\left[d\left(Z_{n}(w),\left[w, Z_{\infty}(w)\right)\right) \geq D, Z_{n}=\gamma\right] \\
& =\sum_{\gamma \in \Gamma} \mathbb{P}\left[d\left(\gamma(w),\left[w, \gamma Z_{n}^{-1} Z_{\infty}(w)\right)\right) \geq D, Z_{n}=\gamma\right] \\
& =\sum_{\gamma \in \Gamma} \mathbb{P}\left[d\left(\gamma(w),\left[w, \gamma Z_{n}^{-1} Z_{\infty}(w)\right)\right) \geq D\right] \mathbb{P}\left[Z_{n}=\gamma\right] \\
& =\sum_{\gamma \in \Gamma} \mathbb{P}\left[d\left(\gamma(w),\left[w, \gamma Z_{\infty}(w)\right)\right) \geq D\right] \mathbb{P}\left[Z_{n}=\gamma\right]
\end{aligned}
$$


The third equality comes from the independence of $Z_{n}=X_{1} X_{2} \cdots X_{n}$ and $Z_{n}^{-1} Z_{\infty}=$ $X_{n+1} X_{n+2} \cdots$. The last equality uses the fact that $Z_{n}^{-1} Z_{\infty}$ and $Z_{\infty}$ have the same law.

On the event $\left\{d\left(\gamma(w),\left[w, \gamma Z_{\infty}(w)\right)\right) \geq D\right\}$, we have in particular $d(w, \gamma(w)) \geq D$ and we can pick $x \in[w, \gamma(w))$ such that $d(\gamma(w), x)=D+O(1)$. Then, because the triangle $\left(w, \gamma(w), \gamma Z_{\infty}(w)\right)$ is thin and since $d\left(\gamma(w),\left[w, \gamma Z_{\infty}(w)\right)\right) \geq D$, we must have $\gamma Z_{\infty}(w) \in$ $\mho_{\gamma(w)}(x, R)$. As usual $R$ is a constant that does not depend on $\gamma, D$ or $Z_{\infty}(w)$. We now apply the lemma of the shadow Lemma 2.8 to the Green metric to deduce that

$\mathbb{P}\left[d\left(\gamma(w),\left[w, \gamma Z_{\infty}(w)\right)\right) \geq D\right] \leq \mathbb{P}\left[\gamma Z_{\infty}(w) \in \mho_{\gamma(w)}(x, R)\right]=\nu_{\gamma}\left(\mho_{\gamma(w)}(x, R)\right) \lesssim e^{-d_{G}(\gamma(w), x)}$.

Finally, using the quasi-isometry between $d$ and $d_{G}$, it follows that

$$
\mathbb{P}\left[d\left(Z_{n}(w),\left[w, Z_{\infty}(w)\right)\right) \geq D\right] \lesssim e^{-b D} .
$$

Proof of (ii). Using the independence of the increments of the walk, one may first assume that $m=0$.

Let us choose $Y_{n}(w) \in\left[w, Z_{\infty}(w)\right)$ such that $d\left(w, Y_{n}(w)\right)$ is as close from $\left(Z_{n}(w) \mid Z_{\infty}(w)\right)$ as possible. Since the space $(X, d)$ is quasiruled, it follows that

$$
d\left(w, Y_{n}(w)\right)=\left(Z_{n}(w) \mid Z_{\infty}(w)\right)+O(1) .
$$

(We only use Landau's notation $O(1)$ for estimates that are uniform with respect to the trajectory of $\left(Z_{n}\right)$. Thus the line just above means that there exists a deterministic constant $C$ such that

$$
\left|d\left(w, Y_{n}(w)\right)-\left(Z_{n}(w) \mid Z_{\infty}(w)\right)\right| \leq C .
$$

The same convention applies to the rest of the proof.)

Let us define

$$
A_{0}=\left\{d\left(w, Y_{n}(w)\right) \leq d\left(w, Y_{n+k}(w)\right)\right\}
$$

and, for $j \geq 1$,

$$
A_{j}=\left\{j-1<d\left(w, Y_{n}(w)\right)-d\left(w, Y_{n+k}(w)\right) \leq j\right\} .
$$

Approximating $\left\{w, Z_{n}(w), Z_{n+k}(w), Z_{\infty}(w)\right\}$ by a tree, it follows that, on the event $A_{0}$,

$$
\left(w \mid Z_{n+k}(w)\right)_{Z_{n}(w)} \leq d\left(Z_{n}(w),\left[w, Z_{\infty}(w)\right)\right)+O(1)
$$

and that, on the event $A_{j}$,

$$
\left(w \mid Z_{n+k}(w)\right)_{Z_{n}(w)} \leq d\left(Z_{n}(w),\left[w, Z_{\infty}(w)\right)\right)+j+O(1)
$$

Therefore

$$
\mathbb{E}\left[\left(w \mid Z_{n+k}(w)\right)_{Z_{n}(w)}\right] \leq \mathbb{E}\left[d\left(Z_{n}(w),\left[w, Z_{\infty}(w)\right)\right)\right]+\sum_{j \geq 1} j \mathbb{P}\left(A_{j}\right)+O(1) .
$$

If $d\left(w, Y_{n}(w)\right)-d\left(w, Y_{n+k}(w)\right) \geq j$ then $d\left(Z_{n+k}(w),\left[Z_{n}(w), Z_{\infty}(w)\right)\right) \geq j$ so that

$$
\mathbb{P}\left(A_{j+1}\right) \leq \mathbb{P}\left[d\left(Z_{n+k}(w),\left[Z_{n}(w), Z_{\infty}(w)\right)\right) \geq j\right] .
$$

Using (i) for the random walk starting at $Z_{n}(w)$, we get

$$
\sum_{j \geq 1} j \mathbb{P}\left(A_{j}\right) \lesssim 1
$$

$4{ }^{\text {e }}$ SÉRIE - TOME $44-2011-\mathrm{N}^{\circ} 4$ 
On the other hand,

$$
\begin{aligned}
\mathbb{E}\left[d\left(Z_{n}(w),\left[w, Z_{\infty}(w)\right)\right)\right] & =\int_{0}^{\infty} \mathbb{P}\left[d\left(Z_{n}(w),\left[w, Z_{\infty}(w)\right)\right) \geq D\right] d D \\
& \lesssim \int_{0}^{\infty} e^{-b D} d D=1 / b .
\end{aligned}
$$

The proposition follows.

We now improve the estimate (2) in Theorem 3.1 when $d_{G} \in \mathscr{D}(\Gamma)$.

COROllary 3.9. - Let $\Gamma$ be a non-elementary hyperbolic group, $(X, d) \in \mathscr{D}(\Gamma)$ and $\mu$ a symmetric law such that $d_{G} \in \mathscr{D}(\Gamma)$, then we have

$$
\limsup \frac{d\left(Z_{n}(w),\left[w, Z_{\infty}(w)\right)\right)}{\log n}<\infty \quad \mathbb{P} \text { a.s. }
$$

Proof. - It follows from Proposition 3.8 that we may find a constant $\kappa>0$ so that

$$
\mathbb{P}\left[d\left(Z_{n}(w),\left[w, Z_{\infty}(w)\right)\right) \geq \kappa \log n\right] \leq \frac{1}{n^{2}} .
$$

Therefore, the Borel-Cantelli lemma implies that

$$
\limsup \frac{d\left(Z_{n}(w),\left[w, Z_{\infty}(w)\right)\right)}{\log n}<\infty \quad \mathbb{P} \text { a.s. }
$$

and the corollary follows.

REMARK. - This corollary was already known in the case of the free group, see [35], and for Brownian motion on a Gromov-hyperbolic manifold and for finite range random walks on hyperbolic graphs [2, Thm 7.3]. After a first version of this paper was publicized, M. Björklund also used the hyperbolicity of the Green metric to prove a Central Limit Theorem for $d_{G}\left(w, Z_{n}(w)\right)$, see [7].

3.5.2. The doubling condition for the harmonic measure. - Let us recall that a measure $m$ is said to be doubling if there exists a constant $C>0$ such that, for any ball $B$ of radius at most the diameter of the space then $m(2 B) \leq C m(B)$.

Proposition 3.10. - Let $\Gamma$ be a non-elementary hyperbolic group, $(X, d) \in \mathscr{D}(\Gamma)$ and let $\mu$ be a symmetric law such that $d_{G} \in \mathscr{D}(\Gamma)$. The harmonic measure is doubling with respect to the visual measure $d_{\varepsilon}$ on $\partial X$.

Proof. - The modern formulation of Efremovich and Tichonirova's Theorem states that quasi-isometries between hyperbolic proper geodesic spaces $\Phi: X \rightarrow Y$ extend as quasisymmetric maps $\phi: \partial X \rightarrow \partial Y$ between their visual boundaries, i.e., there is an increasing homeomorphism $\eta: \mathbb{R}_{+} \rightarrow \mathbb{R}_{+}$such that

$$
|\phi(a)-\phi(b)| \leq \eta(t)|\phi(a)-\phi(c)|
$$

whenever $|a-b| \leq t|a-c|$.

Since $d_{G} \in \mathscr{D}(\Gamma)$, the spaces involved are visual. Thus, the statement remains true since we may still approximate properly the space by trees. 
Since $(X, d)$ and $\left(X, d_{G}\right)$ are quasi-isometric, the boundaries are thus quasisymmetric with respect to $d_{\varepsilon}$ and $d_{\varepsilon}^{G}$. Furthermore, $\nu$ is doubling with respect to $d_{\varepsilon}^{G}$ since it is Ahlforsregular, and this property is preserved under quasisymmetry.

Basic properties of quasisymmetric maps can be found in [21]. More information on boundaries of hyperbolic groups, and the relationships between hyperbolic geometry and conformal geometry can be found in $[11,29]$.

\section{Dimension of the harmonic measure on the boundary of a hyperbolic metric space}

Theorem 1.3 will follow from Proposition 4.1.

We recall the definition of the rates of escape $\ell$ and $\ell_{G}$ of the random walk with respect to $d$ or $d_{G}$ respectively.

$$
\ell \stackrel{\text { def }}{=} \lim _{n} \frac{d\left(w, Z_{n}(w)\right)}{n} \text { and } \ell_{G} \stackrel{\text { def }}{=} \lim _{n} \frac{d_{G}\left(w, Z_{n}(w)\right)}{n} .
$$

We will first prove

Proposition 4.1. - Let $\Gamma$ be a non-elementary hyperbolic group and let $(X, d) \in \mathscr{D}(\Gamma)$. Let $\mu$ be a symmetric probability measure on $\Gamma$ the support of which generates $\Gamma$ such that $d_{G} \in \mathscr{D}(\Gamma)$ and with finite first moment

$$
\sum_{\gamma \in \Gamma} d_{G}(w, \gamma(w)) \mu(\gamma)<\infty
$$

Let $\nu$ be the harmonic measure seen from $w$ on $\partial X$.

For $\nu$-a.e. $a \in \partial X$,

$$
\lim _{r \rightarrow 0} \frac{\log \nu\left(B_{\varepsilon}(a, r)\right)}{\log r}=\frac{\ell_{G}}{\varepsilon \ell},
$$

where $B_{\varepsilon}$ denotes the ball on $\partial X$ for the visual metric $d_{\varepsilon}$.

REMARK. - Recall from [9] that $\mu$ having finite first moment with respect to the Green metric is a consequence of $\mu$ having finite entropy.

Proof. - It is convenient to introduce an auxiliary word metric $d_{w}$ which is of course geodesic. We may then consider the visual quasiruling structure $\mathscr{G}$ induced by the $d_{w}$-geodesics for both metrics $d$ and $d_{G}$.

We combine Propositions 2.1 and 2.6 to get that, for a fixed but large enough $R$, for any $a \in \partial X$ and $x \in[w, a) \subset \mathscr{G}$

$$
B_{\varepsilon}\left(a,(1 / C) e^{-\varepsilon d(w, x)}\right) \subset \mho_{\mathscr{G}}(x, R) \subset B_{\varepsilon}\left(a, C e^{-\varepsilon d(w, x)}\right)
$$

and

$$
B_{\varepsilon}^{G}\left(a,(1 / C) e^{-\varepsilon d_{G}(w, x)}\right) \subset \mho_{\mathscr{G}}(x, R) \subset B_{\varepsilon}^{G}\left(a, C e^{-\varepsilon d_{G}(w, x)}\right)
$$

for some positive constant $C$. We recall that the shadows $\mho_{g}(x, R)$ are defined using geodesics for the word metric $d_{w}$.

The doubling property of $\nu$ with respect to the visual metric $d_{\varepsilon}$ implies that

$$
\nu\left(B_{\varepsilon}\left(a, C e^{-\varepsilon d(w, x)}\right)\right) \asymp \nu\left(\mho_{\mathscr{g}}(x, R)\right)
$$

for any $x \in[w, a)$.

4 e SÉRIE - TOME $44-2011$ - No 4 
Let $\eta>0$; by definition of the drift, there is a set of full measure with respect to the law of the trajectories of the random walk, in which for any sequence $\left(Z_{n}(w)\right)$ and for $n$ large enough, we have $\left|d\left(w, Z_{n}(w)\right)-\ell n\right| \leq \eta n$ and $\left|d_{G}\left(w, Z_{n}(w)\right)-\ell_{G} n\right| \leq \eta n$.

From Theorem 3.1 applied to the metric $d$, we get that, for $n$ large enough, $d\left(Z_{n}(w), \pi_{n}\left(Z_{\infty}(w)\right)\right) \leq \eta n$ and since $d_{G}$ is quasi-isometric to $d$ by assumption, we may also assume that $d_{G}\left(Z_{n}(w), \pi_{n}\left(Z_{\infty}(w)\right)\right) \leq \eta n$.

We conclude that

$$
\left\{\begin{array}{l}
\left|d\left(w, \pi_{n}\left(Z_{\infty}(w)\right)\right)-\ell n\right| \leq 2 \eta n \\
\left|d_{G}\left(w, \pi_{n}\left(Z_{\infty}(w)\right)\right)-\ell_{G} n\right| \leq 2 \eta n
\end{array}\right.
$$

Set

$$
r_{n}=e^{-\varepsilon d\left(w, \pi_{n}\left(Z_{\infty}(w)\right)\right)} .
$$

Therefore, using (7) with $a=Z_{\infty}(w)$ and $x=\pi_{n}\left(Z_{\infty}(w)\right)$, we get

$$
\nu\left(B_{\varepsilon}\left(Z_{\infty}(w), r_{n}\right)\right) \asymp \nu\left(\mho_{\mathscr{G}}\left(\pi_{n}\left(Z_{\infty}(w)\right), R\right)\right) \asymp e^{-d_{G}\left(w, \pi_{n}\left(Z_{\infty}(w)\right)\right)}
$$

where the right-hand part comes from the fact that $\nu$ is a quasiconformal measure of dimension $1 / \varepsilon$ for the Green visual metric and the lemma of the shadow (Lemma 2.8). Hence we deduce from (8) that, if $n$ is large enough, then

$$
\left|\frac{\log \nu\left(B_{\varepsilon}\left(Z_{\infty}(w), r_{n}\right)\right)}{\log r_{n}}-\frac{\ell_{G}}{\varepsilon \ell}\right| \lesssim \eta
$$

Since the measure $\nu$ is doubling (Proposition 3.10), $\nu$ is also $\alpha$-homogeneous for some $\alpha>0$, (cf. [21, Chap. 13]), i.e., there is a constant $C>0$ such that, if $0<r<R<\operatorname{diam} \partial X$ and $a \in \partial X$, then

From

$$
\frac{\nu\left(B_{\varepsilon}(a, R)\right)}{\nu\left(B_{\varepsilon}(a, r)\right)} \leq C\left(\frac{R}{r}\right)^{\alpha} .
$$

it follows that

$$
\left|\log \frac{e^{-\varepsilon n \ell}}{r_{n}}\right| \leq 2 n \varepsilon \eta
$$

Therefore

$$
\left|\log \frac{\nu\left(B_{\varepsilon}\left(Z_{\infty}(w), e^{-\varepsilon n \ell}\right)\right)}{\nu\left(B_{\varepsilon}\left(Z_{\infty}(w), r_{n}\right)\right)}\right| \leq 2 n \alpha \varepsilon \eta+O(1)
$$

$$
\limsup _{n}\left|\frac{\log \nu\left(B_{\varepsilon}\left(Z_{\infty}(w), e^{-\varepsilon n \ell}\right)\right)}{\log e^{-\varepsilon n \ell}}-\frac{\log \nu\left(B_{\varepsilon}\left(Z_{\infty}(w), r_{n}\right)\right)}{\log r_{n}}\right| \lesssim \eta
$$

Since $\eta>0$ is arbitrary, it follows from (9) that

$$
\begin{aligned}
\lim _{r \rightarrow 0} \frac{\log \nu\left(B_{\varepsilon}\left(Z_{\infty}(w), r\right)\right)}{\log r} & =\lim _{n \rightarrow \infty} \frac{\log \nu\left(B_{\varepsilon}\left(Z_{\infty}(w), e^{-\varepsilon n \ell}\right)\right)}{\log e^{-\varepsilon n \ell}} \\
& =\lim _{n \rightarrow \infty} \frac{\log \nu\left(B_{\varepsilon}\left(Z_{\infty}(w), r_{n}\right)\right)}{\log r_{n}}=\frac{\ell_{G}}{\varepsilon \ell} .
\end{aligned}
$$

In other words, for $\nu$ almost every $a \in \partial X$,

$$
\lim _{r \rightarrow 0} \frac{\log \nu\left(B_{\varepsilon}(a, r)\right)}{\log r}=\frac{\ell_{G}}{\varepsilon \ell} .
$$

It remains to prove that $\nu$ has dimension $\ell_{G} / \varepsilon \ell$. This is standard. 


\section{Harmonic measure of maximal dimension}

This section is devoted to the proof of Theorem 1.5 and its corollary.

\subsection{The fundamental equality}

We assume that $d \in \mathscr{D}(\Gamma), \mu$ is a probability measure with exponential moment such that $d_{G} \in \mathscr{D}(\Gamma)$. Thus there exists $\lambda>0$ such that

$$
E \stackrel{\text { def }}{=} \mathbb{E}\left[e^{\lambda d\left(w, Z_{1}(w)\right)}\right]<\infty .
$$

The main issue in the proof of Theorem 1.5 is the following implication which we prove first:

Proposition 5.1. - Under the hypotheses of Theorem 1.5, if $h=\ell v$, then $\rho$ and $\nu$ are equivalent.

Let $R$ be the constant coming from the lemma of the shadow (Lemma 2.8) and write $\mho(x)$ for $\mho(x, R)$.

Let us now define

$$
\varphi_{n}=\frac{\rho\left(\mho\left(Z_{n}(w)\right)\right)}{\nu\left(\mho\left(Z_{n}(w)\right)\right)} \quad \text { and } \quad \phi_{n}=\log \varphi_{n}
$$

Since $\mu^{n}$ is the law of $Z_{n}$, observe that, if $\beta \in(0,1]$, then

$$
\mathbb{E}\left[\varphi_{n}^{\beta}\right]=\sum_{\gamma \in \Gamma} \mu^{n}(\gamma)\left(\frac{\rho(\mho(\gamma(w)))}{\nu(\mho(\gamma(w)))}\right)^{\beta} \text { and } \mathbb{E}\left[\phi_{n}\right]=\sum_{\gamma \in \Gamma} \mu^{n}(\gamma) \log \left(\frac{\rho(\mho(\gamma(w)))}{\nu(\mho(\gamma(w)))}\right)
$$

We start with two lemmata.

Lemma 5.2. - There are finite constants $C_{1} \geq 1$ and $\beta \in(0,1]$ such that, for all $N \geq 1$,

$$
\frac{1}{N} \sum_{1 \leq n \leq N} \mathbb{E}\left[\varphi_{n}^{\beta}\right] \leq C_{1}
$$

When $\mu$ is finitely supported, one can choose $\beta=1$ in the lemma.

Proof. - Let $N \geq 1$ and $1 \leq n \leq N$ be chosen. We will first prove that there are some $\kappa$ and $\beta$ independent from $N$ and $n$ such that

$$
R_{\kappa} \stackrel{\text { def }}{=} \sum_{\gamma, d(w, \gamma(w)) \geq \kappa N}\left(\frac{\rho(\mho(\gamma(w)))}{\nu(\mho(\gamma(w)))}\right)^{\beta} \mu^{n}(\gamma) \lesssim 1 .
$$

We have already seen that the logarithmic volume growth rate for the Green metric is 1 . Then, from the lemma of the shadow (Lemma 2.8) applied to both metrics, we get

$$
\nu(\mho(\gamma(w))) \asymp e^{-d_{G}(w, \gamma(w))}=F(w, \gamma(w)) \asymp G(w, \gamma(w))=\sum_{k} \mu^{k}(\gamma)
$$

and

$$
\rho(\mho(\gamma(w))) \asymp e^{-v d(w, \gamma(w))} .
$$

$4^{\text {e }}$ SÉRIE - TOME $44-2011-\mathrm{N}^{\circ} 4$ 
On the other hand, since $d_{G}$ is quasi-isometric to $d$, it follows that there is a constant $c>0$ such that

$$
\frac{\rho(\mho(\gamma(w)))}{\nu(\mho(\gamma(w)))} \lesssim e^{c d(w, \gamma(w))}
$$

Hence

$$
R_{\kappa} \lesssim \sum_{k \geq \kappa N} e^{c \beta k} \sum_{k \leq d(w, \gamma(w))<k+1} \mu^{n}(\gamma)
$$

But $\mu^{n}$ is the distribution of $Z_{n}$ so that

$$
\sum_{k \leq d(w, \gamma(w))<k+1} \mu^{n}(\gamma) \leq \mathbb{P}\left[d\left(w, Z_{n}(w)\right) \geq k\right] .
$$

From the exponential Tchebychev inequality, one obtains

$$
R_{\kappa} \lesssim \sum_{k \geq \kappa N} e^{(c \beta-\lambda) k} \mathbb{E}\left[e^{\lambda d\left(w, Z_{n}(w)\right)}\right]
$$

Now,

$$
d\left(w, Z_{n}(w)\right) \leq \sum_{0 \leq j<N} d\left(Z_{j}(w), Z_{j+1}(w)\right)=\sum_{0 \leq j<N} d\left(w, Z_{j}^{-1} Z_{j+1}(w)\right)
$$

since $\Gamma$ acts by isometries. Thus, the independence of the increments of the walk implies

$$
\mathbb{E}\left[e^{\lambda d\left(w, Z_{n}(w)\right)}\right] \leq E^{N} .
$$

If we take $\beta \stackrel{\text { def }}{=} \min \{\lambda / 2 c, 1\}$ then (13) becomes

$$
R_{\kappa} \lesssim \sum_{k \geq \kappa N} e^{(-\lambda / 2) k} E^{N} \lesssim e^{-(\lambda / 2) \kappa N} E^{N}
$$

The estimate (10) is obtained by choosing $\kappa=2 \log E / \lambda$.

We now prove that

$$
P_{N} \stackrel{\text { def }}{=} \frac{1}{N} \sum_{1 \leq n \leq N} \sum_{\gamma \in \Gamma: d(w, \gamma(w)) \leq \kappa N}\left(\frac{\rho(\mho(\gamma(w)))}{\nu(\mho(\gamma(w)))}\right)^{\beta} \mu^{n}(\gamma) \lesssim 1 .
$$

Both (10) and (14) imply the lemma.

Note that since $\beta \leq 1$, it follows that $\varphi_{n}^{\beta} \leq \max \left\{1, \varphi_{n}\right\} \leq 1+\varphi_{n}$.

Hence:

$$
\begin{aligned}
P_{N} & \lesssim 1+\frac{1}{N} \sum_{n=1}^{N} \sum_{\gamma \in \Gamma: d(w, \gamma(w)) \leq \kappa N} \frac{\rho(\mho(\gamma(w)))}{\nu(\mho(\gamma(w)))} \mu^{n}(\gamma) \\
& \lesssim 1+\frac{1}{N} \sum_{\gamma \in \Gamma: d(w, \gamma(w)) \leq \kappa N} \frac{\sum_{n=1}^{N} \mu^{n}(\gamma)}{\nu(\mho(\gamma(w)))} \rho(\mho(\gamma(w))) .
\end{aligned}
$$

But (11) implies that

so that

$$
\frac{\sum_{n=1}^{N} \mu^{n}(\gamma)}{\nu(\mho(\gamma(w)))} \lesssim 1
$$

$$
P_{N} \lesssim 1+\frac{1}{N} \sum_{d(w, x) \leq \kappa N} \rho(\mho(x))
$$


Since $\rho(\mho(x)) \asymp e^{-v d(w, x)}$ by (12) and since there are approximately $e^{v k}$ elements in the $d$-ball of radius $k$ (Theorem 2.7 ), we have

$$
\sum_{d(w, x) \leq \kappa N} \rho(\mho(x)) \asymp \sum_{1 \leq n \leq \kappa N} e^{v n} e^{-v n}
$$

and

$$
\sum_{d(w, x) \leq \kappa N} \rho(\mho(x)) \lesssim N
$$

Therefore, the estimate (14) follows from (15).

Lemma 5.3. - There is a finite constant $C_{2} \geq 0$ such that the sequence $\left(\mathbb{E}\left(\phi_{n}\right)+C_{2}\right)_{n \geq 1}$ is subadditive and $(1 / n) \phi_{n}$ tends to $h-\ell v$ a.s. and in expectation.

Proof. - By the lemma of the shadow (Lemma 2.8),

$$
\frac{1}{n} \phi_{n}=\frac{1}{n} d_{G}\left(w, Z_{n}(w)\right)-\frac{1}{n} v d\left(w, Z_{n}(w)\right)+O(1 / n)
$$

so, from Kingman ergodic Theorem it follows that $(1 / n) \phi_{n}$ converges almost surely and in expectation towards

$$
\ell_{G}-\ell v=h-\ell v,
$$

since $h=\ell_{G}$, see [9].

Let $m, n \geq 1$. It also follows from the lemma of the shadow and the triangle inequality for $d_{G}$ that

$$
\begin{aligned}
\mathbb{E}\left[\phi_{m+n}\right]-\left(\mathbb{E}\left[\phi_{m}\right]+\mathbb{E}\left[\phi_{n}\right]\right) & \\
& \leq v \mathbb{E}\left[d\left(w, Z_{m}(w)\right)+d\left(Z_{m}(w), Z_{m+n}(w)\right)-d\left(w, Z_{m+n}(w)\right)\right]+O(1) .
\end{aligned}
$$

So Proposition 3.8 implies the existence of some constant $C_{2}$ such that

$$
\mathbb{E}\left[\phi_{m+n}\right]-\left(\mathbb{E}\left[\phi_{m}\right]+\mathbb{E}\left[\phi_{n}\right]\right) \leq C_{2} .
$$

This gives the desired subadditivity.

Proof of Proposition 5.1. - We shall prove that if $\rho$ and $\nu$ are not equivalent, then $h<\ell v$.

Assuming that $\rho$ and $\nu$ are not equivalent, the ergodicity of both measures implies that $\varphi_{n}$ tends to $0 \mathbb{P}$-a.s.

Choose $\eta \in\left(0, e^{-1}\right]$.

By Egorov theorem, there exist two measurable sets $A$ and $B=A^{c}$ such that $\mathbb{P}[A] \leq \eta$ and $\left(\left.\varphi_{n}\right|_{B}\right)_{n}$ converges uniformly to 0 .

For any $n \geq 1$,

$$
\mathbb{E}\left[\phi_{n}\right]=\int_{A} \phi_{n} d \mathbb{P}+\int_{B} \phi_{n} d \mathbb{P} .
$$

Since $\left(\left.\varphi_{n}\right|_{B}\right)_{n}$ uniformly converges to 0 , there exists $n_{0}$ such that for $n$ larger than $n_{0}$, $\left.\phi_{n}\right|_{B} \leq \log \eta$ and therefore

$$
\int_{B} \phi_{n} d \mathbb{P} \leq \mathbb{P}[B] \log \eta \leq(1-\eta) \log \eta .
$$

Choose $\beta$ and $C_{1}$ as in Lemma 5.2. The Jensen inequality yields

$$
\int_{A} \phi_{n} d \mathbb{P} \leq \frac{\mathbb{P}[A]}{\beta} \log \int_{A} \varphi_{n}^{\beta} \frac{d \mathbb{P}}{\mathbb{P}[A]} \leq \frac{\eta}{\beta} \log (1 / \eta)+\frac{\eta}{\beta} \log ^{+} \mathbb{E}\left[\varphi_{n}^{\beta}\right],
$$

$4^{\text {e }}$ SÉRIE - TOME $44-2011-\mathrm{N}^{\circ} 4$ 
where we have used $\eta \leq 1 / e$.

But Lemma 5.2 implies that $\lim \inf \mathbb{E}\left[\varphi_{n}^{\beta}\right]<2 C_{1}$. So that there exists $p \geq n_{0}$ such that $\mathbb{E}\left[\varphi_{p}^{\beta}\right] \leq 2 C_{1}$.

Hence,

$$
\mathbb{E}\left[\phi_{p}\right] \leq(1-\eta) \log \eta+\frac{\eta}{\beta} \log (1 / \eta)+\frac{\eta}{\beta} \log \left(2 C_{1}\right) .
$$

When $\eta$ tends to 0 , the right-hand side tends to $-\infty$. Therefore, if we fix $\eta$ small enough, there exists $p$ such that

$$
\mathbb{E}\left[\phi_{p}\right]+C_{2} \leq-1,
$$

where $C_{2}$ is the constant appearing in Lemma 5.3.

Lemma 5.3 now implies that

$$
\frac{1}{k}\left(\mathbb{E}\left[\phi_{k p}\right]+C_{2}\right) \leq \mathbb{E}\left[\phi_{p}\right]+C_{2} \leq-1
$$

for $k \geq 1$. As $(1 / p k) \mathbb{E}\left[\phi_{p k}\right]$ tends to $(h-\ell v)$, letting $k$ go to infinity, one obtains

$$
(h-\ell v) \leq \frac{-1}{p}<0 .
$$

RemarK. - In view of the proof of Proposition 5.1, one might wonder whether it is always true that a doubling measure of maximal dimension in an Ahlfors-regular space, as $\nu$ is, has to be equivalent to the Hausdorff measure of the same dimension. This property turns out to be false in general. We are grateful to P. Mattila for pointing out to us its invalidity and to $Y$. Heurteaux for providing us with an explicit example.

\subsection{Equivalent measures}

We let $\Gamma$ be a non-elementary hyperbolic group, $(X, d) \in \mathscr{D}(\Gamma)$, and $\mu$ a probability measure on $\Gamma$ so that $d_{G} \in \mathscr{D}(\Gamma)$. This section is devoted to proving

Proposition 5.4. - If $\rho$ and $\nu$ are equivalent then their density is almost surely bounded, i.e., there is a constant $C \geq 1$ such that for any Borel set $A \subset \partial X$,

$$
\frac{1}{C} \nu(A) \leq \rho(A) \leq C \nu(A) .
$$

We will work with the space $\partial^{2} X$ of distinct points $(a, b) \in \partial X \times \partial X, a \neq b$, which is reminiscent to the geodesic flow of a negatively curved manifold. The group $\Gamma$ acts on $\partial^{2} X$ by the diagonal action $\gamma \cdot(a, b)=(\gamma(a), \gamma(b)), \gamma \in \Gamma$.

We define the following two $\sigma$-finite measures on $\partial^{2} X$ :

$$
d \tilde{\rho}(a, b)=\frac{d \rho(a) \otimes d \rho(b)}{\exp 2 v(a \mid b)} \quad \text { and } \quad d \tilde{\nu}(a, b)=\frac{d \nu(a) \otimes d \nu(b)}{\exp 2(a \mid b)^{G}}
$$

where we define

$$
(a \mid b)^{G \stackrel{\text { def }}{=}} \liminf _{\left(a_{n}\right),\left(b_{n}\right) \rightarrow a, b}\left(a_{n} \mid b_{n}\right)^{G} .
$$

We recall that since $\nu$ is a conformal measure, $\tilde{\nu}$ is invariant, and it is furthermore ergodic [26, Thm 3.3]. On the other hand, $\rho$ being just a quasiconformal measure, it follows that $\tilde{\rho}$ 
is just quasi-invariant, cf. [14]. This implies the existence of a constant $C \geq 1$ such that, for any Borel set $A \subset \partial^{2} X$ and any $\gamma \in \Gamma$,

$$
\frac{1}{C} \tilde{\rho}(A) \leq \tilde{\rho}(\gamma(A)) \leq C \tilde{\rho}(A) .
$$

Proof of Proposition 5.4. - By assumption, there is a positive $\nu$-integrable function $J$ such that $d \rho=J d \nu$. Therefore, $d \tilde{\rho}=\tilde{J} d \tilde{\nu}$ holds with

$$
\tilde{J}(a, b)=J(a) J(b) \frac{\exp 2(a \mid b)^{G}}{\exp 2 v(a \mid b)} .
$$

We shall first prove that $\tilde{J}$ is essentially constant (and non-zero). There is a constant $C>1$ such that the set

$$
A \stackrel{\text { def }}{=}\{(1 / C) \leq \tilde{J} \leq C\}
$$

has positive $\tilde{\nu}$-measure. Since $\tilde{\nu}$ is ergodic, for $\tilde{\nu}$-almost every $(a, b) \in \partial^{2} X$, there exists $\gamma \in \Gamma$ such that $\gamma(a, b) \in A$. It follows from the invariance of $\tilde{\nu}$ and the quasi-invariance of $\tilde{\rho}$ that

$$
\tilde{J}(a, b) \asymp \tilde{J}(\gamma(a), \gamma(b)) .
$$

This proves the claim.

Therefore, for $\tilde{\nu}$-almost every $(a, b)$,

$$
J(a) J(b) \asymp \frac{\exp 2 v(a \mid b)}{\exp 2(a \mid b)^{G}} .
$$

Let us assume that $\log J$ is unbounded in a neighborhood $U$ of a point $a \in \partial X$. We may find a point $b \in \partial X$ with $J(b)$ finite and non-zero, and far enough from $U$ so that

$$
\frac{\exp 2 v(c \mid b)}{\exp 2(c \mid b)^{G}} \asymp 1
$$

for any $c \in U$. This proves that $\log J$ had to be bounded in $U$ : a contradiction.

\subsection{Geometric characterization of the fundamental inequality}

We may now turn to the proof of Theorem 1.5.

Proof of Theorem 1.5. - We first prove that (i), (ii) and (iii) are equivalent. Then we prove that (iii) implies (iv), (iv) implies (v) which implies (iii).

- From Proposition 5.1, we deduce that (i) implies (ii). Proposition 5.4 says that (ii) implies (iii). Furthermore, if $\nu$ and $\rho$ are equivalent, then they have the same Hausdorff dimension. So, from Corollary 1.4 and Theorem 2.7, we get that

$$
\frac{h}{\ell \varepsilon}=\operatorname{dim} \nu=\operatorname{dim} \rho=\frac{v}{\varepsilon},
$$

and thus $h=\ell v$. 
- To prove that (iii) implies (iv), we apply the lemma of the shadow (Lemma 2.8): it follows that, for any $\gamma \in \Gamma$,

$$
e^{-v d(w, \gamma(w))} \asymp \rho(\mho(\gamma(w))) \asymp \nu(\mho(\gamma(w))) \asymp e^{-d_{G}(w, \gamma(w))}
$$

whence the existence of a constant $C$ such that

$$
\left|v d(w, \gamma(w))-d_{G}(w, \gamma(w))\right| \leq C .
$$

Since $\Gamma$ acts transitively by isometries for both metrics, it follows that $(X, v d)$ and $\left(X, d_{G}\right)$ are $(1, C)$-quasi-isometric.

- Assuming (iv), it follows that Busemann functions coincide up to the multiplicative factor $v$. Therefore, the Radon-Nikodym derivative of $\gamma^{*} \nu$ with respect to $\nu$ at a point $a \in \partial X$ is proportional to $\exp \left(-v \beta_{a}\left(w, \gamma^{-1}(w)\right)\right)$ a.e. Therefore, $\nu$ is a quasiconformal measure for $\left(\partial X, d_{\varepsilon}\right)$. This is $(\mathrm{v})$.

- For the last implication, (v) implies (iii), one can use the uniqueness statement in Theorem 2.7 to get that $\rho$ and $\nu$ are equivalent and have bounded density. This proves (iii).

\subsection{Simultaneous random walks}

We now turn to the proof of Corollary 1.6.

Proof of Corollary 1.6. - Let us consider the Green metric $d_{G}$ associated with $\mu$ and denote by $\widehat{\ell}$ the drift of $\left(\widehat{Z}_{n}\right)$ in the metric space $\left(\Gamma, d_{G}\right)$. Theorem 1.1 implies that $d_{G} \in \mathscr{D}(\Gamma)$.

Assumption (i) translates into $\widehat{h}=\widehat{\ell}$. Since $v_{G}=1$, this means that $\widehat{\nu}$ has maximal dimension in the boundary of $\left(\Gamma, d_{G}\right)$ endowed with a visual metric. Therefore Theorem 1.5 implies the equivalence between (i) and (iii).

Exchanging the roles of $\mu$ and $\widehat{\mu}$ gives the equivalence between (ii) and (iii).

If $\widehat{d}_{G}$ denotes the Green metric for $\widehat{\mu}$, then (iv) means that $d_{G}$ and $\widehat{d}_{G}$ are $(1, C)$-quasiisometric, which is equivalent to (iii) by Theorem 1.5.

\subsection{Fuchsian groups with cusps}

The next proposition is the key to the proof of Theorem 1.10. Let us first introduce its setting.

Let $X$ be a proper quasiruled hyperbolic space and let $\Gamma$ be a hyperbolic subgroup of isometries that acts properly discontinuously on $X$. Consider a symmetric probability measure $\mu$ on $\Gamma$ with finite support and whose support generates the group $\Gamma$. Let $\nu$ be the corresponding harmonic measure on $\partial \Gamma$, the visual boundary of $\Gamma$.

Let $\Gamma(w)$ be the orbit of some point $w \in X$. As for Theorem 3.1, Theorem 7.3 in [28] and $\S 7.4$ therein imply that the sequence $Z_{n}(w)$ almost surely converges to some point $Z_{\infty}(w)$ in $\partial X$, the visual boundary of $X$. Let $\nu_{1}$ be the law of $Z_{\infty}(w)$.

Although the two spaces $\partial X$ and $\partial \Gamma$ might be topologically different, the two measured spaces $\left(\partial X, \nu_{1}\right)$ and $(\partial \Gamma, \nu)$ are isomorphic as $\Gamma$-spaces, i.e., there exists a measured spaces isomorphism $\Phi$ from $(\partial \Gamma, \nu)$ to $\left(\partial X, \nu_{1}\right)$ that conjugates the action of $\Gamma$ on both spaces. Indeed both spaces are models for the Poisson boundary of the random walk. This is proved in [28] Theorem 7.7 and Remark 3 following it for $\left(\partial X, \nu_{1}\right)$ and it is a general fact for the Martin boundary $(\partial \Gamma, \nu)$. 
Proposition 5.5. - Let $X$ be a proper quasiruled hyperbolic space endowed with a geometric group action. Let $\rho$ be the corresponding Patterson-Sullivan measure. Let $\Gamma$ be a hyperbolic subgroup of isometries that acts properly discontinuously on $X$ and $\Gamma(w)$ be an orbit of $\Gamma$ in $X$. Let $\mu$ be a symmetric probability measure on $\Gamma$ with finite support and whose support generates the group $\Gamma$. Let $\nu_{1}$ be the limit law of the trajectories of the random walk on $\partial X$. If $\rho$ and $\nu_{1}$ are equivalent then $\Gamma$ and $X$ are quasi-isometric.

Proof. - One checks as in Proposition 5.4 that, once $\rho$ and $\nu_{1}$ are equivalent, then their density is almost surely bounded.

We recall the following change of variables formula:

$$
\frac{d \gamma^{*} \nu}{d \nu}(a)=K_{a}\left(\gamma^{-1}\right)
$$

for $\nu$ almost any point $a \in \partial \Gamma$ and where $K_{a}$ is the Martin kernel. Because of the isomorphism $\Phi$, we also have

for $\nu_{1}$ almost any point $\xi \in \partial X$.

$$
\frac{d \gamma^{*} \nu_{1}}{d \nu_{1}}(\xi)=K_{\Phi^{-1}(\xi)}\left(\gamma^{-1}\right)
$$

On the other hand, $\rho$ being a quasiconformal measure, it satisfies

$$
\frac{d \gamma^{*} \rho}{d \rho}(\xi) \asymp e^{v \beta_{\xi}\left(w, \gamma^{-1}(w)\right)},
$$

where $\beta_{\xi}$ is the Busemann function in $X$.

Since the density of $\nu_{1}$ with respect to $\rho$ is bounded and bounded away from 0 , we therefore have

$$
K_{\Phi^{-1}(\xi)}\left(\gamma^{-1}\right) \asymp e^{v \beta_{\xi}\left(w, \gamma^{-1}(w)\right)}
$$

for $\rho$ almost any $\xi$.

We now use Lemma 2.5. First observe that, given $x, y \in X, \sup _{\xi \in \partial X} \beta_{\xi}(x, y)$ can be replaced by an essential sup with respect to $\rho$ since $\rho$, being quasiconformal, charges any non empty ball and since $\xi \rightarrow \beta_{\xi}(x, y)$ is locally almost constant. So we get from Lemma 2.5 that

$$
\left|d(x, y)-\operatorname{ess} \sup _{\xi \in \partial X} \beta_{\xi}(x, y)\right|
$$

is bounded. By a similar argument, applying Lemma 2.5 to the Green metric on $\Gamma$, we deduce that

$$
\mid d_{G}\left(e, \gamma^{-1}\right)-\text { ess } \sup _{\xi \in \partial \Gamma} \log K_{\xi}\left(\gamma^{-1}\right) \mid
$$

is bounded. The essential sup is taken with respect to $\nu$.

But (16) implies that

$$
\mid \text { ess } \sup _{a \in \partial \Gamma} \log K_{a}\left(\gamma^{-1}\right)-v \text { ess } \sup _{\xi \in \partial X} \beta_{\xi}\left(w, \gamma^{-1}(w)\right) \mid
$$

is bounded and therefore

$$
\sup _{\gamma \in \Gamma}\left|d_{G}\left(e, \gamma^{-1}\right)-v d\left(w, \gamma^{-1}(w)\right)\right|<\infty .
$$

We conclude that $\Gamma$ and $\Gamma(w)$ are quasi-isometric, so that the action of $\Gamma$ on $X$ is quasiconvex. But, by assumption, its limit set is the whole boundary $\partial X$, hence $\Gamma$ and $X$ are quasiisometric.

$4^{\text {e }}$ SÉRIE - TOME $44-2011-$ No $^{\circ}$ 
Proof of Theorem 1.10. - We proceed by contradiction and assume that $\nu_{1}$ is equivalent to the Lebesgue measure $\lambda$ on $\mathbb{S}^{1}$.

First note that we can restrict our attention to the subgroup generated by the support of $\mu$. If this subgroup turned out to have infinite covolume then its boundary would be a strict subset of $\mathbb{S}^{1}$ and $\nu_{1}$ would certainly not be equivalent to the Lebesgue measure. Therefore we may, and will, assume that the support of $\mu$ generates $\Gamma$ and that $\Gamma$ has finite covolume and is finitely generated.

We know from Selberg's lemma that $\Gamma$ contains a torsion-free subgroup $\Gamma_{S}$ of finite index so that $\mathbb{H}^{2} / \Gamma_{S}$ is a compact Riemann surface with finitely many punctures. Therefore $\Gamma_{S}$ is isomorphic to a free group so that $\Gamma$ is hyperbolic and its boundary is a perfect, totally disconnected, compact set (a Cantor set). But Proposition 5.5 implies that $\Gamma$ should be quasiisometric to $\mathbb{H}^{2}$. As a consequence the boundary of $\Gamma$ would be homeomorphic to the unit circle, the contradiction we were looking for.

\section{Discretisation of Brownian motion}

We let $M$ be the universal covering of a Riemannian manifold $N$ of pinched negative curvature and finite volume with deck transformation group $\Gamma$, i.e., $M / \Gamma=N$. We let $d$ denote the distance defined by the Riemannian structure on $M$. Note that when $N$ is compact, $\Gamma$ acts geometrically on $M$, and since it has negative curvature, it follows that $\Gamma$ is hyperbolic and that $M$ is quasi-isometric to $\Gamma$ by Švarc-Milnor's lemma.

\subsection{The discretised motion}

W. Ballmann and F. Ledrappier have refined a method of T. Lyons and D. Sullivan [37], further studied by A. Ancona [2], V. Kaimanovich [25], and by A. Karlsson and F. Ledrappier [30] which replaces the Brownian motion $\left(\xi_{t}\right)$ by a random walk which shadows its paths [5]. More precisely, we have [4, 5, 25, 30, 37]:

THEOREM 6.1. - There exists a probability measure $\mu$ on $\Gamma$ such that its associated random walk $\left(Z_{n}\right)$ satisfies the following properties:

(i) The measure $\mu$ is symmetric with full support but has a finite first moment with respect to d. If $N$ is compact then $\mu$ has a finite exponential moment.

(ii) There exists a positive constant $T$ such that, almost surely and in $L^{1}$,

$$
\lim \frac{d\left(\xi_{k T}, Z_{k}(w)\right)}{k}=0 .
$$

(iii) The Green function $G_{\mu}$ of the random walk is proportional to the Green function $G_{M}$ of $M$.

(iv) The harmonic measures for the Brownian motion and the random walk on $\partial M$ coincide.

We are able to prove the following: 
THEOREM 6.2. - Under the notation and assumptions from above, let $d_{G}$ denote the Green metric associated with $\mu$. If $N$ is compact, then $d_{G} \in \mathscr{D}(\Gamma)$ and

$$
\operatorname{dim} \nu=\frac{h_{M}}{\varepsilon \ell_{M}}
$$

where $h_{M}$ and $\ell_{M}$ denote the entropy and the drift of the Brownian motion respectively.

Proof. - The acronyms (ED) and (QR) below refer to Proposition 3.5. Since $M$ has pinched negative curvature, it follows that $G_{M}(x, y) \lesssim e^{-c d(x, y)}$ holds for some constant $c>0$, see [3, (2.4) p. 434]. By part (iii) of Theorem 6.1, $G_{\mu}$ and $G_{M}$ are proportional. Therefore $G_{\mu}$ also satisfies $G_{\mu}(x, y) \lesssim e^{-c d(x, y)}$ and (ED) is proved. Furthermore, A. Ancona's Theorem 3.7 also holds for the Brownian motion, see [2], showing that (QR) holds as well. Both these properties imply that $\left(X, d_{G}\right) \in \mathscr{D}(\Gamma)$ by Proposition 3.5.

The identity $h_{\mu}=h_{M} \cdot T$ was proved by V. Kaimanovich [23, 25]. Furthermore, A. Karlsson and F. Ledrappier have established $\ell_{\mu}=\ell_{M} \cdot T$ [30]. Thus, Corollary 1.4 implies the dimension formula.

\subsection{Examples}

Let us fix $n \geq 2$ and consider the hyperbolic space $\mathbb{H}^{n}$ of constant sectional curvature -1 . The explicit form of the Green function on this space shows easily that, given $w, x, y, z \in \mathbb{H}^{n}$ which are at distance $c>0$ apart from one another, one has

$$
\Theta(x, y) \gtrsim \min \{\Theta(x, z), \Theta(z, y)\}
$$

where $\Theta$ is Naïm's kernel, and the implicit constant depends only on $c$. Let $N$ be a finite volume hyperbolic manifold with deck transformation group $\Gamma$ acting on $\mathbb{H}^{n}$. The estimate (17) shows that the Green metric $d_{G}$ on $\Gamma$ associated with the discretized Brownian motion on $\mathbb{H}^{n}$ is hyperbolic. Moreover, the estimate (ED) holds as well, so that the Green metric $d_{G}$ is quasi-isometric to the restriction of the hyperbolic metric to the orbit $\Gamma(o)$ of a base point $o \in \mathbb{H}^{n}$. Since $N$ has finite volume, the limit set of $\Gamma$ is the whole sphere at infinity, and it coincides with the visual boundary of $\left(\Gamma, d_{G}\right)$. Therefore, Theorem 1.7 implies that the Martin boundary coincides with $\partial \mathbb{H}^{n}$, homeomorphic to $\mathbb{S}^{n-1}$. We omit the details.

We apply this construction in two special cases.

If we consider for $N$ a punctured 2-torus with a complete hyperbolic metric of finite volume (as in [5]), we obtain an example of a random walk on the free group for which the Green metric is hyperbolic but its boundary $\mathbb{S}^{1}$ does not coincide with the boundary of the group (which is a Cantor set). Therefore, $d_{G}$ does not belong to the quasi-isometry class of the free group.

If we consider now for $N$ a complete hyperbolic 3-manifold of finite volume with a rank 2 cusp, then its fundamental group is not hyperbolic since it contains a subgroup isomorphic to $\mathbb{Z}^{2}$, but the Green metric is hyperbolic nonetheless.

4 e SÉRIE - TOME $44-2011-$ No $^{\circ}$ 


\section{REFERENCES}

[1] A. Ancona, Positive harmonic functions and hyperbolicity, in Potential theorysurveys and problems (Prague, 1987), Lecture Notes in Math. 1344, Springer, 1988, $1-23$.

[2] A. Ancona, Théorie du potentiel sur les graphes et les variétés, in École d'été de Probabilités de Saint-Flour XVIII-1988, Lecture Notes in Math. 1427, Springer, 1990, 1-112.

[3] M. T. Anderson, R. Schoen, Positive harmonic functions on complete manifolds of negative curvature, Ann. of Math. 121 (1985), 429-461.

[4] W. Ballmann, On the Dirichlet problem at infinity for manifolds of nonpositive curvature, Forum Math. 1 (1989), 201-213.

[5] W. Ballmann, F. Ledrappier, Discretization of positive harmonic functions on Riemannian manifolds and Martin boundary, in Actes de la Table Ronde de Géométrie Différentielle (Luminy, 1992), Sémin. Congr. 1, Soc. Math. France, 1996, 77-92.

[6] G. Besson, G. Courtois, S. Gallot, Entropies et rigidités des espaces localement symétriques de courbure strictement négative, Geom. Funct. Anal. 5 (1995), 731799.

[7] M. BJöRkLund, Central limit theorems for Gromov hyperbolic groups, J. Theoret. Probab. 23 (2010), 871-887.

[8] S. BlaChÈre, S. BrofFERIO, Internal diffusion limited aggregation on discrete groups having exponential growth, Probab. Theory Related Fields 137 (2007), 323-343.

[9] S. Blachère, P. Haïssinsky, P. Mathieu, Asymptotic entropy and Green speed for random walks on countable groups, Ann. Probab. 36 (2008), 1134-1152.

[10] M. Bonk, O. Schramm, Embeddings of Gromov hyperbolic spaces, Geom. Funct. Anal. 10 (2000), 266-306.

[11] M. Bourdon, H. Pajot, Quasi-conformal geometry and hyperbolic geometry, in Rigidity in dynamics and geometry (Cambridge, 2000), Springer, 2002, 1-17.

[12] R. Bowen, C. Series, Markov maps associated with Fuchsian groups, Publ. Math. I.H.É.S. 50 (1979), 153-170.

[13] C. Connell, R. Muchnik, Harmonicity of quasiconformal measures and Poisson boundaries of hyperbolic spaces, Geom. Funct. Anal. 17 (2007), 707-769.

[14] M. Coornaert, Mesures de Patterson-Sullivan sur le bord d'un espace hyperbolique au sens de Gromov, Pacific J. Math. 159 (1993), 241-270.

[15] B. Deroin, V. Kleptsyn, A. Navas, On the question of ergodicity for minimal group actions on the circle, Mosc. Math. J. 9 (2009), 263-303.

[16] E. B. Dynkin, The boundary theory of Markov processes (discrete case), Uspehi Mat. Nauk 24 (1969), 3-42.

[17] E. Ghys, P. De LA HAR Pe (eds.), Sur les groupes hyperboliques d'après Mikhael Gromov, Progress in Math. 83, Birkhäuser, 1990.

[18] Y. GuivarC'H, Sur la loi des grands nombres et le rayon spectral d'une marche aléatoire, in Conference on Random Walks (Kleebach, 1979), Astérisque 74, Soc. Math. France, 1980, 47-98. 
[19] Y. Guivarc'h, Y. Le Jan, Sur l'enroulement du flot géodésique, C. R. Acad. Sci. Paris Sér. I Math. 311 (1990), 645-648.

[20] Y. Guivarc'H, Y. Le Jan, Asymptotic winding of the geodesic flow on modular surfaces and continued fractions, Ann. Sci. École Norm. Sup. 26 (1993), 23-50.

[21] J. Heinonen, Lectures on analysis on metric spaces, Universitext, Springer, 2001.

[22] G. A. Hunt, Markoff chains and Martin boundaries, Illinois J. Math. 4 (1960), 313340.

[23] V. A. KaĬmanovich, Brownian motion and harmonic functions on covering manifolds. An entropic approach, Soviet Math. Dokl. 33 (1986), 812-816.

[24] V. A. Kaimanovich, Invariant measures of the geodesic flow and measures at infinity on negatively curved manifolds, Hyperbolic behaviour of dynamical systems, Ann. Inst. H. Poincaré Phys. Théor. 53 (1990), 361-393.

[25] V. A. Kaimanovich, Discretization of bounded harmonic functions on Riemannian manifolds and entropy, in Potential theory (Nagoya, 1990), de Gruyter, 1992, 213 223.

[26] V. A. Kaimanovich, Ergodicity of harmonic invariant measures for the geodesic flow on hyperbolic spaces, J. reine angew. Math. 455 (1994), 57-103.

[27] V. A. Kaimanovich, Hausdorff dimension of the harmonic measure on trees, Ergodic Theory Dynam. Systems 18 (1998), 631-660.

[28] V. A. Kaimanovich, The Poisson formula for groups with hyperbolic properties, Ann. of Math. 152 (2000), 659-692.

[29] I. Kapovich, N. Benakli, Boundaries of hyperbolic groups, in Combinatorial and geometric group theory (New York, 2000/Hoboken, NJ, 2001), Contemp. Math. 296, Amer. Math. Soc., 2002, 39-93.

[30] A. Karlsson, F. Ledrappier, Propriété de Liouville et vitesse de fuite du mouvement brownien, C. R. Math. Acad. Sci. Paris 344 (2007), 685-690.

[31] V. Le Prince, Dimensional properties of the harmonic measure for a random walk on a hyperbolic group, Trans. Amer. Math. Soc. 359 (2007), 2881-2898.

[32] V. Le Prince, A relation between dimension of the harmonic measure, entropy and drift for a random walk on a hyperbolic space, Electron. Commun. Probab. 13(2008), $45-53$.

[33] F. Ledrappier, Ergodic properties of Brownian motion on covers of compact negatively-curve manifolds, Bol. Soc. Brasil. Mat. 19 (1988), 115-140.

[34] F. Ledrappier, Harmonic measures and Bowen-Margulis measures, Israel J. Math. 71 (1990), 275-287.

[35] F. LEDRAPPIER, Some asymptotic properties of random walks on free groups, in Topics in probability and Lie groups: boundary theory, CRM Proc. Lecture Notes 28, Amer. Math. Soc., 2001, 117-152.

[36] R. LyOns, Equivalence of boundary measures on covering trees of finite graphs, Ergodic Theory Dynam. Systems 14 (1994), 575-597.

[37] T. Lyons, D. Sullivan, Function theory, random paths and covering spaces, J. Differential Geom. 19 (1984), 299-323.

$4^{\mathrm{e}}$ SÉRIE - TOME $44-2011-\mathrm{N}^{\circ} 4$ 
[38] J. Mairesse, F. Mathéus, Random walks on free products of cyclic groups, J. Lond. Math. Soc. 75 (2007), 47-66.

[39] L. Naï̀, Sur le rôle de la frontière de R. S. Martin dans la théorie du potentiel, Ann. Inst. Fourier, Grenoble 7 (1957), 183-281.

[40] M. A. Pinsky, Stochastic Riemannian geometry, in Probabilistic analysis and related topics, Vol. 1, Academic Press, 1978, 199-236.

[41] J.-J. PrAT, Étude asymptotique et convergence angulaire du mouvement brownien sur une variété à courbure négative, C. R. Acad. Sci. Paris Sér. A-B 280 (1975), AA1539A1542.

[42] J. VÄIsÄLÄ, Gromov hyperbolic spaces, Expo. Math. 23 (2005), 187-231.

[43] A. M. Vershik, Dynamic theory of growth in groups: entropy, boundaries, examples, Russian Math. Surveys 55 (2000), 667-733.

[44] W. Woess, Random walks on infinite graphs and groups, Cambridge Tracts in Mathematics 138, Cambridge Univ. Press, 2000.

[45] L. S. Young, Dimension, entropy and Lyapunov exponents, Ergodic Theory Dynam. Systems 2 (1982), 109-124.

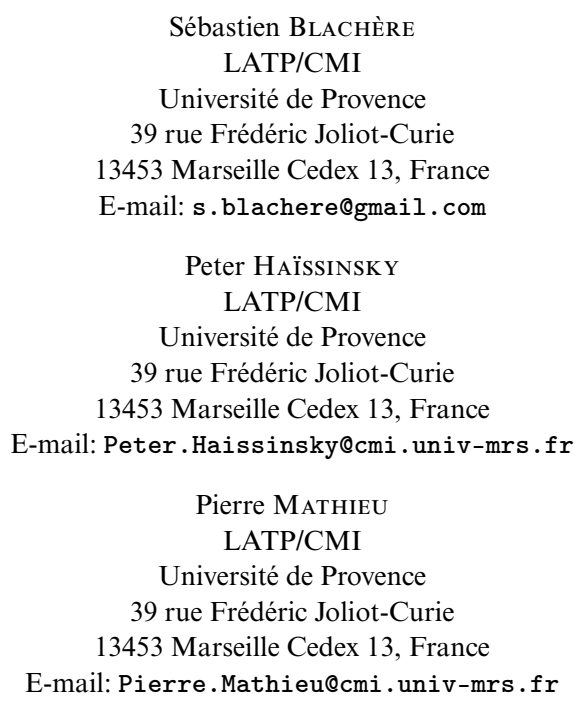

